\title{
A Location-Procurement-Distribution Model Combined with Data Envelopment Analysis Model under a Multi-period Environment and Gradual Budget Injection for Humanitarian Relief Supply Chains
}

Leyla Fazli ( $\sim$ leyla.fazli@yahoo.com )

Ferdowsi University of Mashhad Faculty of Engineering

Original Research

Keywords: Humanitarian relief logistics, Location-procurement-distribution problem, Dynamic model, Human suffering, Data envelopment analysis model.

Posted Date: February 3rd, 2021

DOI: https://doi.org/10.21203/rs.3.rs-180144/v1

License: (c) (i) This work is licensed under a Creative Commons Attribution 4.0 International License. Read Full License 


\section{A Location-Procurement-}

Distribution Model Combined with

Data Envelopment Analysis Model under a Multi-period Environment and Gradual Budget Injection for Humanitarian Relief Supply Chains

\section{Leyla Fazli}

Ph.D. Student, Department of Industrial Engineering, Ferdowsi University of Mashhad, Mashhad, Iran, Tel. +98-9372283193, Email: leyla.fazli@yahoo.com. 


\title{
A Location-Procurement-Distribution Model Combined with Data Envelopment Analysis Model under a Multi-period Environment and Gradual Budget Injection for Humanitarian Relief Supply Chains
}

\begin{abstract}
Humanmade or natural catastrophes such as droughts, floods, earthquakes, storms, coups, economic and political crises, wars, and so forth impact various areas of the world annually. Furthermore, the lack of adequate preparations and proper coping against them causes nations to suffer heavy losses and casualties, which are sometimes irrecoverable. Consequently, as an essential activity in crisis management, humanitarian relief logistics has been of particular importance and has taken a good deal of notice at the international level during recent years. Aid facilities location and the storage of necessary commodities before a disaster and the proper distribution of relief commodities among demand points following a disaster are critical logistical strategies to improve performance and reduce latency when responding to a given disaster. In this regard, this study presents a stochastic multi-objective mixed-integer non-linear programming model in a two-level network that includes warehouses and affected areas. The model aims at minimizing total social costs, which include the expense of founding warehouses, the expense of procuring commodities, and deprivation cost, as well as maximizing fulfilled demands and warehouses utility. In this study, several pre-disaster periods, a limited budget for establishing warehouses and procuring relief commodities with their gradual injection into the system, the time value of money, various criteria for evaluating warehouses, the risk of disruption in warehouses and transportation networks, and heterogeneous warehouses are considered. The maximization of warehouses utility is done according to a data envelopment analysis model. Moreover, a multi-objective fuzzy programming model called the weighted max-min model is applied to solve the proposed model. Ultimately, the outcomes of the evaluation and validation of the proposed model show its appropriate and efficient performance.
\end{abstract}

Keywords: Humanitarian relief logistics, Location-procurement-distribution problem, Dynamic model, Human suffering, Data envelopment analysis model. 


\section{Introduction}

Disasters have always significantly and extensively impacted and threatened human lives all around the world. However, humans are still unable to predict accurately and prevent disasters. Typically, factors such as increased unprincipled urbanization, climate change, terrorism, and human and animal diseases, which are simultaneously associated with more human activity on the earth, provide the basis for the occurrence of various disasters such as floods, hurricanes, earthquakes, droughts, and so forth. On the one hand, the increase in the number of catastrophes and the expansion of their destructive range, and on the other hand, population growth in different areas of the world have increased material losses and human casualties caused by such incidents. Despite technological advances, calamities and problems caused by disasters have always been accounted for as one of the major obstacles against the sustainable development of countries. Figs. 1-4 show the number of occurrences, the deaths, and the affected population, as well as economic losses globally by disaster type between 2008 and 2017 and 2018, respectively (EM-DAT ${ }^{1}$, 2019).

The losses caused by disasters cannot be compensated in various aspects, especially the human aspect; however, with preventive measures and proper planning to provide necessary preparations for coping with such incidents, these losses can be reduced as much as possible. Additionally, since the severity and dimensions of disasters are often extensive, the volume of the demand created for rescue operations is very high after a disaster occurs. Moreover, the relief centers, which can meet the requirements under normal conditions, are often not enough to respond to the

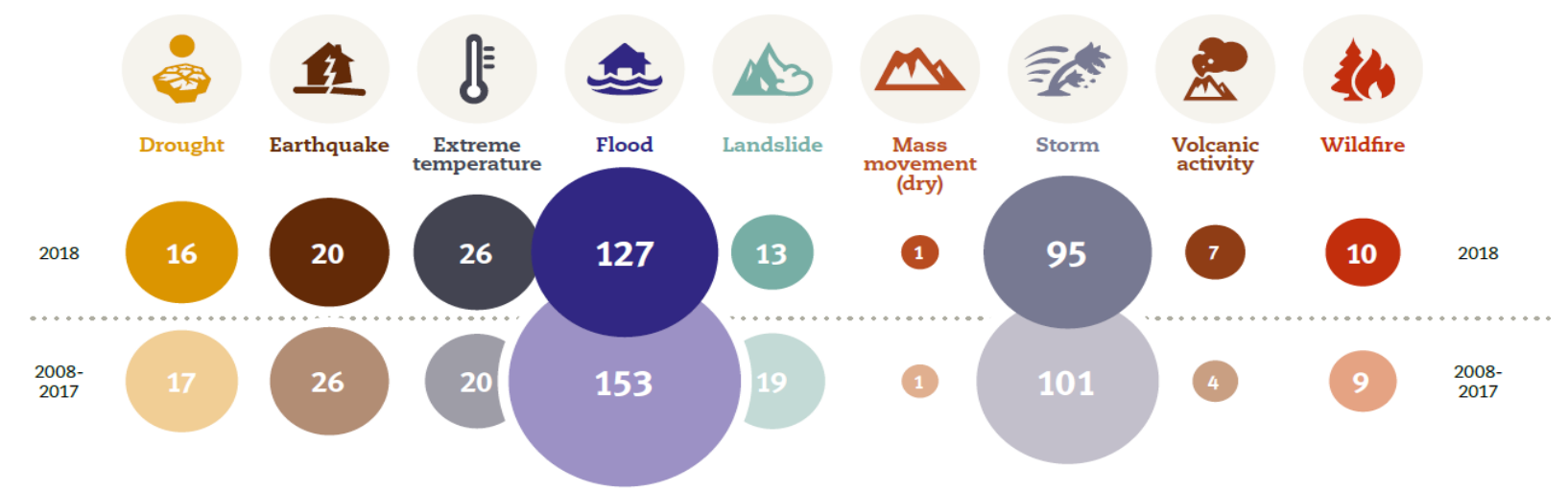

Fig. 1. The number of occurrences globally by disaster type between 2008 and 2017 and in 2018.

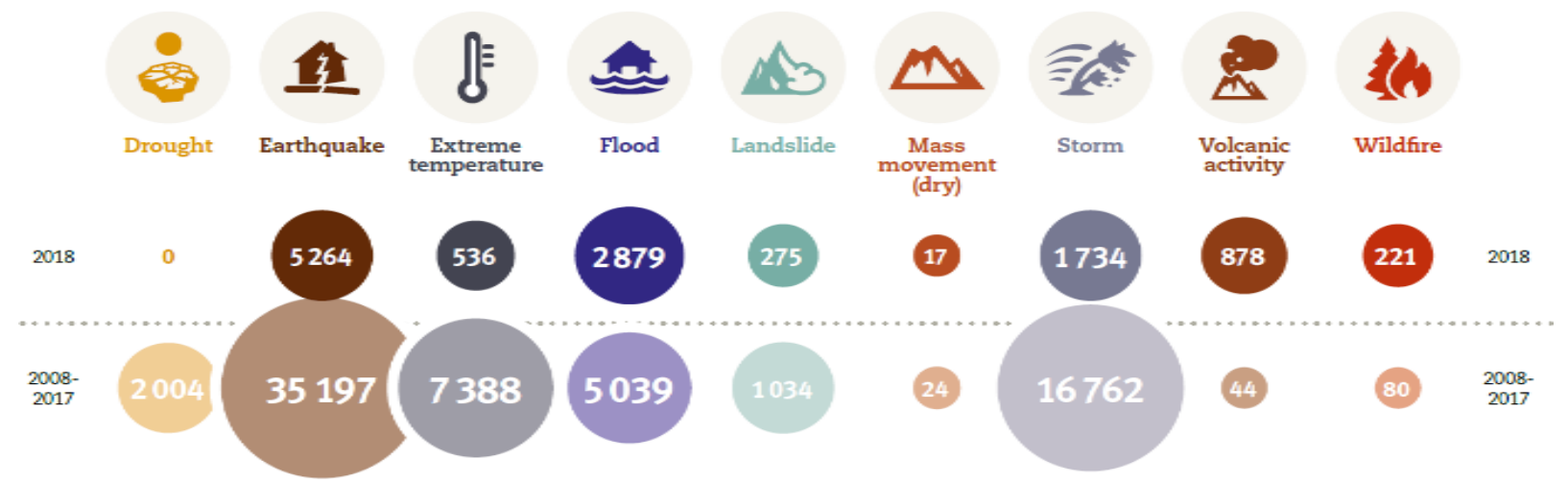

Fig. 2. The number of deaths globally by disaster type between 2008 and 2017 and in 2018.

\footnotetext{
1. www.emdat.be
} 


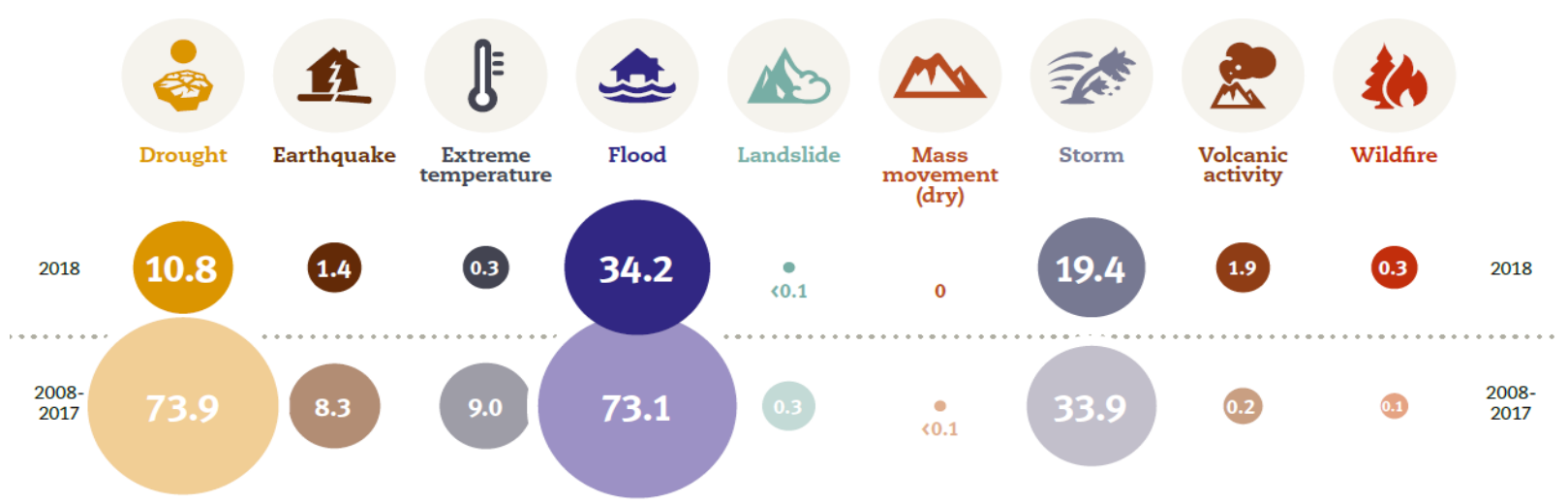

Fig. 3. The number of affected (million) globally by disaster type between 2008 and 2017 and in 2018.

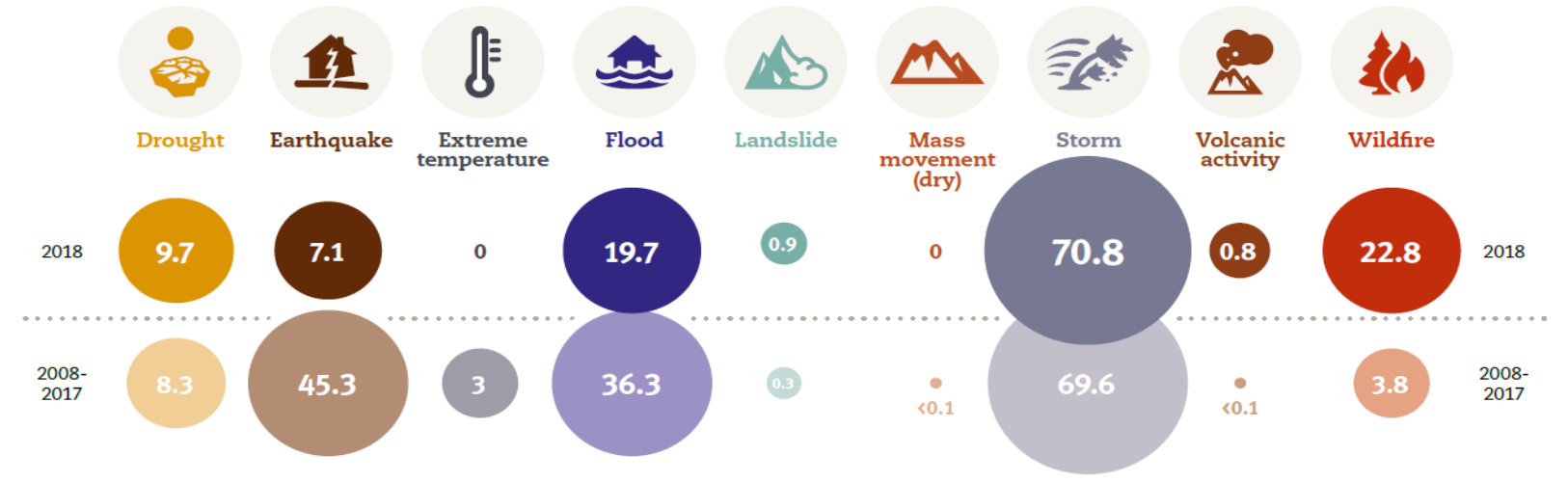

Fig. 4. Economic losses (billion US\$) globally by disaster type between 2008 and 2017 and in 2018.

demand generated under these conditions quickly. Importantly, actions such as handling affected people, sending essential items, providing first aids, and transporting injured people to medical centers at the right time, especially within the first 72 hours following the occurrence of disasters (Relief Golden Hours), play a significant role in decreasing casualties and disabilities caused by disasters (Jang, Lien and Tsai, 2009). In such complicated emergencies, decision-makers must respond to logistical problems quickly and effectively, which will not be possible except through careful, systematic, pre-designed, and improved planning for coping with disaster.

Therefore, this study aims to design a relief logistics network to quickly and efficiently respond to the requirements generated by the crisis, increase the productivity of existing facilities, and reduce losses and costs.

The rest of the study is structured as follows: The general definition of the problem under examination and the main motivations and contributions behind the study are explained in section 2. Some of the research related to this study are reviewed in section 3 . The proposed mathematical programming model and its solution algorithm are described in section 4. A numerical example along with the performance evaluation of the model is presented in section 5. Finally, concluding statements and recommendations for future studies are expressed in section 6.

\section{General Definition of the Problem}

The occurrence of disasters necessitates the provision of reliefs including individuals, materials and items, equipment, resources, and financial assistance, and all other assistance that are 
dispatched to reduce damages and casualties in each affected area; as a result, these reliefs need to be managed. The 1980s can be known as when crisis management began flourishing as one of the most critical and significant issues globally (Thomas and Kopczak, 2005).

The diversity of communities' needs, goals, capabilities, and limitations has led to the presentation of different definitions for crisis management. The process of predicting and preventing a crisis, encountering and intervening in a crisis, and recovering after a crisis is called crisis management (İlhan, 2011). There are different approaches to determining crisis management phases. One of these approaches, which most researchers agree on, classifies crisis management phases into four categories, including mitigation, preparedness, response, and recovery (Altay and Green Iii, 2006).

Given the practical experiences gained by managing catastrophic crises globally, the presence of supply chains and logistics experts in crisis management has become necessary. It has also created a research branch called humanitarian relief logistics. Humanitarian relief logistics is a part of logistics that deals with the preparedness and response phases in a crisis management system. Humanitarian relief logistics has been defined as follows:

The process of planning, performing, and controlling efficiency, cost flow, and storage of commodities considering the information from the start spot to the consumption spot to reduce losses and people's injuries encompasses activities such as preparedness, planning, procurement, warehousing, transportation, tracking and tracing, and customs clearance (Thomas and Kopczak, 2005).

Humanitarian relief logistics usually requires procurement, storage, human resources, machinery and equipment, and transportation of food, water, medicine, and so forth. It also involves various activities both before and after the crisis. Facility location, inventory management, network flow, routing, scheduling, evacuation of people, and emergency settlement are among major activities in humanitarian relief logistics. Therefore, due to the variety of logistics operations in disaster relief, the operation is a considerably expensive one; accordingly, humanitarian relief logistics is the most costly part of disaster aid operation (about 80\%) (Tomasini and Van Wassenhove, 2009). Furthermore, in relief operations, many vehicles, tools, and human resources need to be arranged. For example, in the Wenchuan earthquake on May $12^{\text {th }}, 2008$, in China, nineteen helicopters, six transport planes, about 5800 relief workers, and 150 tons of equipment were dispatched to the affected area within 24 hours.

The structure of humanitarian relief logistics consists of three main steps: 1 . The use of suppliers and procurement; 2. Predetermined location for distribution and medical centers and warehousing; 3. Transportation (Tomasini and Van Wassenhove, 2009). Fig. 5 shows the humanitarian relief supply chain structure that encompasses these three steps (Li, Ramshani and Huang, 2018). The first step in any humanitarian relief logistics structure refers to supplying the required equipment for people in affected areas. Every relief organization needs to provide essential items via suppliers using methods such as direct purchase and bidding. The most significant efforts in this step include reducing purchase costs (considering the probabilistic inflation after the disaster), ensuring that items are available on time, reducing delivery time, and coordinating the donated items with the required items in affected areas. (Balcik and Beamon, 2008).

After preparing required goods and equipment for periods before and after disasters, relief agencies must transport them to predetermined places such as distribution centers and warehouses for storage. In this step, efforts are made to reduce the high expenses for establishing warehouses and distribution centers, and the costs of maintaining commodities (The possible damage to the 
commodities should also be considered.). Besides, there is a high risk of damage to the warehouse during disasters. Therefore, suitable locations should be selected among candidate points for warehouses and distribution centers.

In the end, transportation is the last critical step in the structure of humanitarian relief logistics in which goods and equipment are transferred to distribution centers, intermediate distribution points, medical centers, and finally, the affected areas. Post-disaster transportation is one of the most challenging steps in this structure, even if various preventive measures are considered (Beamon and Balcik, 2008). These are challenging conditions since transportation equipment and facilities are usually subject to damage. Moreover, weather conditions and lack of security in affected areas may limit the type of vehicles and how to use them.

Due to the unpredictable nature of crises and the myriad of damages caused by them, humanitarian relief logistics has created a critical and vital background within the discussion of disaster relief operations and it represents a vital and critical lever for improving cost, quality, and time in this discussion. The shortage of relief goods in affected spots leads to increased human losses. Therefore, designing and developing an effective strategy for expediting the transfer of relief goods to affected spots is very important in coping with disasters.

The proper location for stocking relief goods pre-disaster, effective planning for preparing relief goods both before and after the disaster, and designing an efficient network for distributing relief items among demand points can be suggested as a solution. These strategies can reduce response time and the costs associated with it, and any incorrect measures in these areas lead to a dramatic increase in human and financial losses. Besides, lack of attention to the interactions among crisis management phases can be a considerable obstacle against useful reactions to a crisis. Optimizing the activities of these phases separately cannot necessarily lead to the optimization of the entire relief and rescue operation; it may sometimes lead to impractical decisions in crises. As a result, integrating the crisis management phases in issues related to relief logistics network optimization is of necessity. Subsequently, due to the growing importance of crisis management in today's world, this study plans two phases of preparedness and response by modeling decisions related to warehouses location, the storage capacity, retrofitting level of each warehouse, the

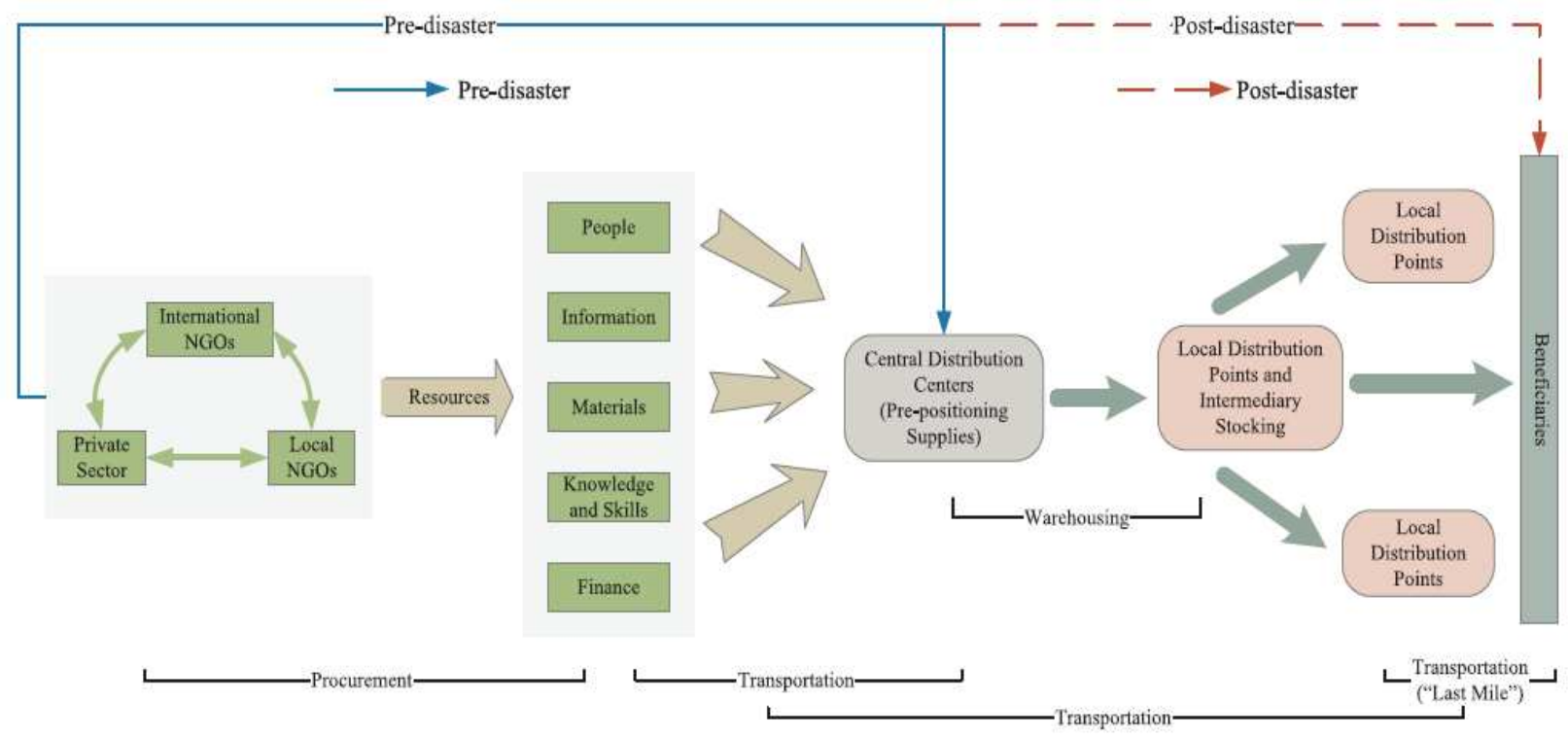

Fig. 5. Humanitarian relief supply chain structure. 
procurement of relief goods pre-disaster, and how to give out relief items among the disaster victims post-disaster in the form of an integrated mathematical programming model assuming real conditions. The following are the main innovations of this study in the background of humanitarian relief logistics:

- Despite the large number of research conducted in humanitarian relief logistics, real conditions are overlooked in many inquiries as a result of the complexity of the problem. For example, budget constraints in planning are only considered in a few studies in this field. Moreover, budget injection into a system in these studies is taken into account in instantaneously (i.e., full access to the required budgets at the beginning of a planning time horizon). Meanwhile, budgets can be gradually made available over time (gradual budget injection) in the real world in addition to budget limitations; subsequently, budget constraints in establishment and procurement along with gradual budget injection into the system pre-disaster are considered in the present study. Accordingly, given the assumed financial constraints, several pre-disaster periods are considered, and procurement, and distribution problems are modeled dynamically (time-dependent) and simultaneously. It is noteworthy that in the research conducted on humanitarian relief logistics, the necessary relief facilities are established simultaneously predisaster; as a result, the location problem is modeled as static (time-independent). However, in real conditions, the simultaneous establishment of the required relief facilities pre-disaster cannot be possible due to various reasons such as shortage of financial resources, lack of human resources, and so forth.

- A new objective function is presented, which maximizes fulfilled demands and minimizes deprivation costs ${ }^{1}$ while simultaneously considering the priority of demand points and equitable responsiveness or distribution ${ }^{2}$. This objective function is calculated in terms of the fraction of fulfilled demand, the reverse deprivation cost function proposed by Holguín-Veras et al. (2016), and the priority of demand point.

- To locate the required relief facilities using mathematical programming models in crisis management, the only criteria used include storage capacity, travel time, cost, and disruption risk. In addition to these criteria, other criteria are also considered in this study, and warehouses utility based on these criteria is maximized. Considering the specific features and benefits of DEA models, the maximization of warehouses utility is formulated in the proposed programming model according to a proposed DEA model according to the DEA model introduced by Sun et al. (2013). In this DEA model (Sun et al., 2013), the efficiency of alternatives (decision-making units (DMUs)) can only be evaluated by minimizing the summation of the weighted coordinate distances between a virtual ideal alternative and all alternatives. Nevertheless, in addition to minimizing the summation of these weighted

\footnotetext{
1. Deprivation costs include the costs imposed on casualties due to lack of access to required items or services (Holguín-Veras et al., 2013); consequently, these costs represent human suffering. Given the importance of deprivation costs in humanitarian relief logistics, certain researchers including Holguín-Veras have focused on how to estimate deprivation cost for each person affected by a disaster and have presented different deprivation cost functions.

${ }^{2}$. The concept of equity and how to measure it have been widely investigated in the literature. Braveman and Gruskin (2003) defined equity as the lack of systematic disparities among groups of people. The consideration of the equity concept in allocation/distribution decisions represents supplying demand points in a fair manner as well as the best efforts to ensure that the required relief commodities are equally distributed among all demand points. Equitable relief distribution among demand points is also an important point in humanitarian relief logistics (Beamon and Balcik, 2008). The three main approaches frequently used to achieve equity as an objective in relief distribution include: 1. Minimax approach; 2. Maximin approach; and 3. Maxisum approach (Ransikarbum and Mason, 2016).
} 
coordinate distances in this study, the maximum weighted coordinate distances between the virtual ideal alternative and all alternatives are also minimized. Interestingly, the concepts of DEA models are integrated with mathematical programming models in few studies so far (e.g., Klimberg and Ratick (2008) and Afsharian (2019)). The rest of these studies are also similar to Klimberg and Ratick's research (2008). Therefore, it can be concluded that the DEA model presented by Sun et al. (2013) or its developed models have not been integrated with mathematical programming models to date.

o In addition to the above cases, this study involves other innovations as well. For instance, the time value of money is also considered in the study as the funds used are invested in a bank with a specific interest rate; as a result, the remaining establishment and procurement budgets at the end of a period are transferred to the next period while the interest rate of the period is applied.

\section{Literature review}

Investigation on humanitarian relief logistics began by the operations research community in the 1990s (Van Hentenryck, Bent and Coffrin, 2010). However, the growing trend in crises has attracted increased attention to humanitarian relief logistics in recent years; accordingly, many studies have been conducted in this field so far. The following are some research, which have simultaneously planned both preparedness and response phases and have focused on the problems discussed in this study (relief facilities location, procurement, and distribution of relief goods) within a network of humanitarian relief logistics, including a set of relief facilities (distribution centers, warehouses, transfer points and shelters) and demand points (injured areas, shelters and health, and medical centers):

In the studies conducted by $\mathrm{Lu}$ (2013), Lu and Sheu (2013), Bell, Fonzone and Polyzoni (2014), Salman and Yücel (2015), Mohamadi and Yaghoubi (2017), Noham and Tzur (2018), Rahmani et al. (2018), Mohamadi and Yaghoubi and Pishvaee (2019), the proper location of aid facilities was determined in the preparedness step, as well as the distribution strategy of required goods was set in the response step. Lu and Sheu (2013) proposed a robust vertex p-center model. Lu (2013) extended his previous model ( $\mathrm{Lu}$ and Sheu (2013)) by considering the uncertainty in demand in addition to travel time and prioritizing demand points based on their demands. Bell et al. (2014) presented a capacitated p-median model and estimated the best-worst travel time between each warehouse-demand point pair using a game theory approach under the worst-case probabilities of link failure. Salman and Yücel (2015) considered both dependent and independent link failures. The outcomes indicate that dependent link failure significantly improves fulfilled demand percentages in comparison with independent link failure. In order to cope with disruption risks at the main distribution centers, Mohamadi and Yaghoubi (2017), Rahmani et al. (2018), and Mohamadi et al. (2019) used backup distribution centers. Mohamadi and Yaghoubi (2017) also planned for transferring the injured and determined the amount of response time violation for curing the injured considering the triage system. To locate main distribution centers, the probabilistic set-covering problem presented by Hwang (2004) was used. Rahmani et al. (2018) presented a stochastic programming model according to the robust scenario-based optimization approach presented by Mulvey, Vanderbei and Zenios (1995). Mohamadi et al. (2019) also focused on people's settlement management and presented a stochastic multi-objective programming model according to the maximal covering model presented by Daskin and Dean (2005). In the research conducted by Noham and Tzur (2018), the decision-maker also decides to schedule a response to every affected area. In this study, each affected area is devoted to the nearest 
distribution center, and inventory is distributed among demand points fairly and equitably by presenting a new constraint.

Balcik and Beamon (2008), Mete and Zabinsky (2010), Irohara, Kuo and Leung (2013), Rath and Gutjahr (2014), Rath and Gutjahr (2014), Bozorgi-Amiri and Khorsi (2016), Caunhye et al. (2016), Rezaei-Malek et al. (2016a), Rezaei-Malek et al. (2016b), Condeixa et al. (2017), Elçi and Noyan (2018), Li, Ramshani and Huang (2018), Tavana et al. (2018), Torabi et al. (2018), Cotes and Cantillo (2019), Hu and Dong (2019), Noyan, Meraklı and Küçükyavuz (2019), Wang and Nie (2019), Chen (2020) focused on pre-disaster location and procurement problems as well as a distribution problem. Balcik and Beamon (2008) determined distribution centers location, the amounts of relief commodities stored at each center, and the share of each center in satisfying demands considering a criticality weight for each relief item, different coverage levels for each commodity with different priorities, and satisfying the larger amount of demand at the lower coverage level, in a network including a set of distribution centers and a demand point. Elçi and Noyan (2018), Noyan et al. (2019), and Wang and Nie (2019) also decided on the size (storage capacity) of distribution centers. Noyan et al. (2019) presented a stochastic risk-averse programming model by considering a constraint according to the risk measure multi-criteria conditional value-at-risk (CVAR). Elçi and Noyan (2018) presented a chance-constrained meanrisk stochastic model according to CVAR. Also, in this study, lost demand is only possible in certain scenarios where the sum of the probabilities of their occurrences is less than or equal to a certain amount. Wang and Nie (2019) considered traffic congestion and a criticality weight for each relief item. A multi-level optimization approach was adopted in the studies conducted by Mete and Zabinsky (2010), Irohara et al. (2013), Caunhye et al. (2016), Condeixa et al. (2017), and Chen (2020). Mete and Zabinsky (2010) first determined the locations for storing medical supplies, the amount of storage of each supply, and how to distribute each supply among hospitals using a two-level stochastic optimization approach. Then, they investigated the loading and routing problems of vehicles in the response phase by presenting another single-level programming model and the results obtained via the previous model. Irohara et al. (2013) proposed a three-level programming model. The first level addresses pre-disaster location, procurement, and allocation decisions; the second level indicates damages caused by the disaster (the distribution centers that may be destroyed by the disaster), while the third level determines decisions were related to the response and recovery phases (new distribution centers location, distribution of supplies, and the recovery of destroyed distribution centers). Caunhye et al. (2016) proposed a stochastic two-level programming model and focused on inventory management, routing, and fleet management problems in the response phase as well. Condeixa et al. (2017) focused on how to procure relief goods both pre-disaster and post-disaster and presented a stochastic two-level risk-averse programming model according to CVAR. The obtained outcomes demonstrate that risk aversion can lead to better decisions. Chen (2020) first proposed a stochastic two-level programming model with known demand distributions. Then, assuming that demand ranges within a certain interval, he reformulated the model into another model called the risk-averse $\Psi$-expander model. Renkli and Duran (2014) first presented a deterministic programming model, followed by developing the following model in two ways: 1 . by considering the blockage probability of the path between a relief facility and a demand point; subsequently, they introduced and implemented a chance constraint for each demand point and commodity, which ensures reliability for a demand point to receive its relief goods, into the formulation; 2 . by considering different disaster scenarios. The obtained results indicate that the major impact of chance constraints is that relief facilities are located outside of risky areas. Hence, chance constraints provide minimum reliability for relief 
good transportation and outperform the formulations without chance constraints in terms of the expected unfulfilled demand. Nevertheless, chance constraints increase the average distance. In another study, Rezaei-Malek et al. (2016a) considered human suffering by maximizing a new measure called the utility level of the delivered relief commodities. This measure simultaneously takes into account the priorities of different relief goods and affected areas, the importance of delivery time, and the fraction of the covered demand at each affected area. In this study, the equitable distribution of goods along with determining transportation routes and required vehicles were examined. Li et al. (2018) presented a cooperative maximal covering model where relief items were prioritized. In the studies conducted by Rath et al. (2014), Bozorgi-Amiri and Khorsi (2016), Torabi et al. (2018), Cotes and Cantillo (2019), and Hu and Dong (2019), a multi-sourcing problem ${ }^{1}$ was used to procure relief items. Rath and Gutjahr (2014) formulated the distribution of relief items as a routing problem and also determined the optimal budget for pre-disaster operative costs. Bozorgi-Amiri and Khorsi (2016) took several periods in the response phase into account. They also decided on the size of distribution centers, the procurement of relief goods, routing between the different levels of the network, and transport fleet management at each period while considering the priorities of different relief commodities and affected areas, backordered demand for certain items, and lost demand for other items. Torabi et al. (2018) also focused on the postdisaster procurement of relief items and the size of central depots. Furthermore, they combined a multi-sourcing problem with a quantity flexibility contract. Cotes and Cantillo (2019) also decided on how to procure relief items in the response phase. They minimized human suffering by minimizing the total social cost $^{2}$. Hu and Dong (2019) paid attention to the post-disaster procurement of necessary goods and the size of distribution centers. Price discounts based on delivery time and order quantity, as well as physical inventory were taken into account as supplier selection criteria. Additionally, it was assumed that the supplies purchased from suppliers after a disaster are either being produced by suppliers or sent from the suppliers' warehouses (their physical inventory). Since suppliers' physical inventory is originally planned to serve their regular customers, the relief organization would pay a fine to suppliers for using their physical inventory to compensate for the risk of losing these customers. Rezaei-Malek et al. (2016b) and Tavana et al. (2018) investigated the inventory management of perishable goods with specific expiration dates at different periods before a disaster occurs. Rezaei-Malek et al. (2016b) considered equitable distribution of medical items among hospitals and proposed a stochastic two-objective programming model according to the robust scenario-based optimization approach proposed by Mulvey et al. (1995) and $\mathrm{Yu}$ and $\mathrm{Li}$ (2000). The obtained results show that the robust scenariobased stochastic optimization approach leads to a significant cost saving compared to the scenariobased stochastic approach. Moreover, this research was carried out by Tavana et al. in 2018 by considering a set of affected areas in addition to warehouses and hospitals and modeling the distribution of relief items within the network in the form of a multi-echelon multi-depot vehicle routing problem.

Aiming at efficient responsiveness and the cost-effective procurement of the necessary relief items, Balcik and Ak (2014) considered a supplier selection problem in which agreements are established with selected suppliers. They focused on a quantity flexibility contract where the aid agency commits to purchasing a minimum of a total quantity from each selected supplier after the disaster occurs and over an agreed period; in return, the suppliers reserve their capacity for the

\footnotetext{
1. It is a special case of supplier selection problem, which determines which suppliers should be selected and how much should be purchased from each selected supplier.

${ }^{2}$. Total social cost includes both logistics costs and deprivation costs.
} 
agency and commit to delivering supplies based their contract. Also, to procure relief items in both phases of preparedness and response, Aghajani, Torabi, and Heydari (2020) integrated a new twoperiod option contract with inventory prepositioning and supplier selection.

In a three-level relief logistics network including supply nodes, transitional nodes, and demand nodes, Liberatore et al. (2014) determined the storage quantity of a required relief commodity at each supply node both in pre-disaster and post-disaster steps; they also adjusted the commodity flow within the network and arrival time at each network node as well as deciding on the repairing of damaged routes. In this study, equitable distribution along with the reliability and safety of the distribution routes were considered as well.

Zhang et al. (2019) considered the probability of a disaster occurring after a primary disaster (secondary disaster) and estimated this probability as a conditional probability that depends on the primary disaster. They proposed a stochastic three-level multi-objective programming model in line with distributing relief supplies among the primary and secondary disaster points, managing the post-disaster transport fleet, and determining whether relief supplies should be stocked at the centers before a disaster. The obtained results show that sustainable rescue ability can be considerably improved by considering secondary disasters solely relative to primary disasters.

Wang et al. (2020) proposed a stochastic programming model to determine the locations and the capacities of warehouses in the preparedness phase and procure and distribute relief commodities in the response phase. Accordingly, a seismic resilience function was introduced to measure the earthquake resistance of the demand point. Furthermore, analytic hierarchy process, fault tree analysis, neural network, and fuzzy evaluation were applied to calculate the value of seismic resilience of each affected area.

Table 1 summarizes the body of research examined above and presents the major differences between these studies and the present paper.

\section{Statement of the Problem}

This research aims to present a model, which includes decisions in phases of preparedness and response in line with managing the crisis relief supply chain. Subsequently, this study focuses on relief facilities location, inventory planning and management, and distribution problems. Predisaster warehouse location and inventory management affect the performance of relief activities as the number and locations of warehouses and the amount of relief commodities stored in them straightly affect demand coverage, response time, and the expenses incurred all over the relief supply chain. The general outline of the desired humanitarian supply chain is presented in Fig. 6. In this research, it is hypothesized that several types of relief items must be stored in warehouses pre-disaster; then following the disaster, these relief items must be transported from storage warehouses to the affected areas (demand points). Thus, decision-makers would decide on the optimal location of warehouses as well as their storage capacity and retrofitting level, the quantities of relief items purchased for each warehouse, and how to distribute relief items among demand points over a pre-disaster multi-period time interval.

In the following subsections, the hypotheses of the proposed model, the introduction of the indices, parameters, and decision variables applied in it, and its formulation and solution algorithm are presented.

\section{1. Hypotheses}

The main hypotheses considered regarding the problem under consideration are as follows: 
- Relief items are packaged in certain numbers in packages and each relief package is allocated to an affected person.

○ Demand points do not have the same response priority; therefore, a preference score is assigned to each demand point.

○ There are different disaster scenarios.

○ Demand, amount of warehouse inventory that remains usable after the disaster, and travel time are scenarios-based stochastic parameters.

- There are limited and specific budgets for establishing warehouses and procuring relief goods. Also, the total establishment and the procurement budget are not readily available at the beginning of the planning time horizon; nonetheless, budgets become available gradually, during the time planning horizon (gradual budgets injection into the system). Therefore, several pre-disaster periods are considered. In addition, the injection time of each part of the budgets into the system is at the beginning of periods.

- Since the funds used are invested with a certain interest rate, the remaining of the establishment and procurement budgets at the end of a period are transferred to its next period while considering the interest rate of the period.

Table 1. A comparison among the proposed model and the models presented in the above research.

\begin{tabular}{|c|c|c|c|c|c|c|c|c|c|}
\hline References & $\begin{array}{l}\text { Multi- } \\
\text { Product }\end{array}$ & $\begin{array}{l}\text { Multi- } \\
\text { Period }\end{array}$ & $\begin{array}{c}\text { Risks of } \\
\text { Disruption }\end{array}$ & $\begin{array}{l}\text { Resilience } \\
\text { Strategies }\end{array}$ & $\begin{array}{c}\text { Shortage } \\
\text { of Product }\end{array}$ & Uncertainty & $\begin{array}{l}\text { Dynamic } \\
\text { Location }\end{array}$ & $\begin{array}{l}\text { Equitable } \\
\text { Response }\end{array}$ & $\begin{array}{l}\text { Number } \\
\text { of Models }\end{array}$ \\
\hline Balcik and Beamon (2008) & $\checkmark$ & & & & LD & SS & & & 1 \\
\hline Mete and Zabinsky (2010) & $\checkmark$ & & TR & & LD & SS & & & 2 \\
\hline Irohara et al. (2013) & & & $\mathrm{F}$ & LT,RF & & & & & 1 \\
\hline Rath and Gutjahr (2014) & & & & & LD & & & & 1 \\
\hline Renkli and Duran (2014) & $\checkmark$ & & TR & & & Model3: SS & & & 3 \\
\hline $\begin{array}{l}\text { Bozorgi-Amiri and } \\
\text { Khorsi (2016) }\end{array}$ & $\checkmark$ & A & $\mathrm{F}, \mathrm{V}, \mathrm{TR}$ & & $\mathrm{LD}, \mathrm{BD}$ & $\mathrm{SS}$ & & $\checkmark$ & 1 \\
\hline Caunhye et al. (2016) & & & TR & LT & LD & SS & & & 1 \\
\hline $\begin{array}{l}\text { Rezaei-Malek et al. } \\
\text { (2016a) }\end{array}$ & $\checkmark$ & & $\mathrm{F}, \mathrm{TR}$ & & LD & $\mathrm{SS}$ & & $\checkmark$ & 1 \\
\hline $\begin{array}{l}\text { Rezaei-Malek et al. } \\
(2016 b)\end{array}$ & $\checkmark$ & $\mathrm{B}$ & $\mathrm{F}, \mathrm{TR}$ & & LD & SS & & $\checkmark$ & 1 \\
\hline Condeixa et al. (2017) & $\checkmark$ & & $\mathrm{F}, \mathrm{TR}$ & & LD & SS & & & 1 \\
\hline Elçi and Noyan (2018) & $\checkmark$ & & $\mathrm{F}, \mathrm{TR}$ & & LD & SS & & & 1 \\
\hline Li et al. (2018) & $\checkmark$ & & & & LD & SS & & & 1 \\
\hline Tavana et al. (2018) & $\checkmark$ & $\mathrm{B}$ & $\mathrm{F}$ & & LD & & & & 1 \\
\hline Torabi et al. (2018) & $\checkmark$ & & & & LD & $\mathrm{SS}, \mathrm{F}, \mathrm{M}$ & & & 1 \\
\hline Cotes and Cantillo (2019) & $\checkmark$ & & & & & & & & 1 \\
\hline $\mathrm{Hu}$ and Dong (2019) & & & $\mathrm{V}, \mathrm{F}$ & & LD & SS & & & 1 \\
\hline Noyan et al. (2019) & $\checkmark$ & & $\mathrm{F}$ & $\mathrm{LT}$ & LD & SS & & $\checkmark$ & 1 \\
\hline Wang and Nie (2019) & $\checkmark$ & & $\mathrm{F}, \mathrm{TR}$ & LT & LD & SS & & & 2 \\
\hline Chen et al. (2020) & $\checkmark$ & & & & LD & $\begin{array}{l}\text { Model1: SS } \\
\text { Model2: R }\end{array}$ & & & 2 \\
\hline Present Work & $\checkmark$ & $\mathrm{B}$ & $\mathrm{F}, \mathrm{TR}$ & FF & LD & $\mathrm{SS}$ & $\checkmark$ & $\checkmark$ & 1 \\
\hline
\end{tabular}


Table 1 (continued)

\begin{tabular}{|c|c|c|c|c|}
\hline References & Programming Models & $\begin{array}{l}\text { Objective } \\
\text { Functions }\end{array}$ & $\begin{array}{c}\text { Budget } \\
\text { Constraints }\end{array}$ & $\begin{array}{l}\text { Meta-Heuristic } \\
\text { Algorithm }\end{array}$ \\
\hline Balcik and Beamon (2008) & MIP,LP & DC & IB & \\
\hline Mete and Zabinsky (2010) & Model 1: MLP,MIP,LP; Model 2: MIP,LP & C,T,DC & & \\
\hline Irohara et al. (2013) & MLP,MIP,LP & $\mathrm{C}$ & & $\checkmark$ \\
\hline Rath and Gutjahr (2014) & MOP,MIP,LP & $\mathrm{C}, \mathrm{DC}, \mathrm{O}$ & IB & $\checkmark$ \\
\hline Renkli and Duran (2014) & $\begin{array}{c}\text { Model 1: MIP,LP; Model 2: MIP,NLP; } \\
\text { Model 3: MIP,LP }\end{array}$ & $\mathrm{D}$ & & \\
\hline Bozorgi-Amiri and Khorsi (2016) & MOP,MIP,NLP & $\mathrm{C}, \mathrm{T}, \mathrm{E}$ & & \\
\hline Caunhye et al. (2016) & MLP,MIP,NLP & $\mathrm{C}, \mathrm{T}, \mathrm{DC}, \mathrm{E}$ & & \\
\hline Rezaei-Malek et al. (2016a) & MOP,MIP,NLP & $\mathrm{C}, \mathrm{E}, \mathrm{U}$ & & $\checkmark$ \\
\hline Rezaei-Malek et al. (2016b) & MOP,MIP,LP & $\mathrm{C}, \mathrm{T}$ & IB & \\
\hline Condeixa et al. (2017) & MLP,MIP,LP & $\mathrm{C}, \mathrm{RI}$ & & \\
\hline Elçi and Noyan (2018) & MIP,LP & $\mathrm{C}, \mathrm{RI}$ & & \\
\hline Li et al. (2018) & MIP,LP & DC & IB & \\
\hline Tavana et al. (2018) & MOP,MIP,LP & $\mathrm{C}, \mathrm{T}$ & IB & $\checkmark$ \\
\hline Torabi et al. (2018) & MIP,LP & $\mathrm{C}$ & IB & $\checkmark$ \\
\hline Cotes and Cantillo (2019) & MIP,NLP & $\mathrm{C}$ & & \\
\hline Hu and Dong (2019) & MIP,LP & $\mathrm{C}$ & & \\
\hline Noyan et al. (2019) & MIP,LP & $\mathrm{C}$ & & \\
\hline Wang and Nie (2019) & Model 1: MIP,NLP; Model 2: MIP,LP & $\mathrm{C}$ & & \\
\hline Chen et al. (2020) & Model 1: MLP,MIP,LP; Model 2: MIP,NLP & $\mathrm{C}$ & & $\checkmark$ \\
\hline Present Work & MOP,MIP,NLP & C,DC,E,U & GB & \\
\hline
\end{tabular}

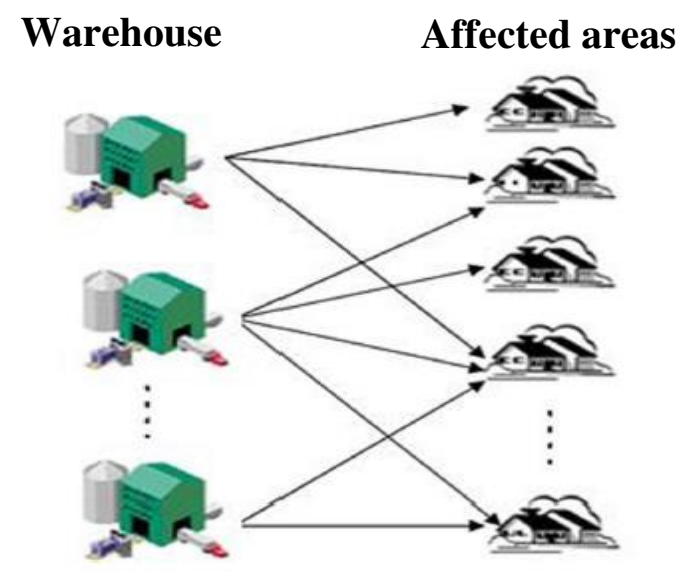

Fig. 6. The general outline of the proposed humanitarian relief supply chain.

- An irrecoverable shortage of relief items at demand points is possible (lost demand).

- Since the storage capacity and retrofitting levels of warehouses can be different, they are not homogeneous.

O The costs of establishing warehouses and procuring relief packages and bank interest rate depend on time and other time-independent parameters.

$\circ$ The risk of disruption in the transportation network is taken into account in travel time. 
- A disaster can disrupt the capability of warehouses by damaging them. Therefore, the risk of disruption in warehouses is taken into account by considering the amount of warehouse inventory that remains usable after the disaster.

- To specify the proper location of warehouses in addition to criteria including travel time, storage capacity, establishment cost, procurement cost, and disruption risk, other criteria are also used. These criteria are evaluated by a proposed Data Envelopment Analysis (DEA) model according to the DEA model introduced by Sun et al. (2013), which is integrated with the proposed mathematical programming model. Subsequently, these criteria are divided into two categories of input and output criteria ${ }^{1}$.

o The deprivation cost function according to the function presented by Holguín-Veritas et al. (2016) is considered as follows:

$F(t)=0.9814 e^{0.0188 t}$

Where $t$ is travel time.

- Warehouses are established in such a way that they can be operational at the beginning of the period or shortly after. Similarly, access to the ordered relief items and their storage is possible at the beginning of the period or shortly after.

\section{2. Sets, Indices, Parameters, and Decision Variables of the Proposed Model}

In order to present the mathematical programming model more properly, first the required symbols, parameters, and variables are defined:

\section{Sets and indices:}

I: Set of candidate sites for establishing warehouses, indexed by $i$.

$J$ : Set of demand points, indexed by $j$.

$T$ : Set of periods, indexed by $t, t$.

$S$ : Set of scenarios, indexed by $s$.

$L$ : Set of the storage capacity levels of warehouses, indexed by $l$.

$R$ : Set of the retrofitting levels of warehouses, indexed by $r$.

$N$ : Set of inputs criteria, indexed by $n$.

$M$ : Set of outputs criteria, indexed by $m$.

\section{Parameters:}

$p c_{i t}$ : Cost of procuring a relief package for warehouse $i$ at period $t$.

$f_{\text {ilrt }}$ : Cost of establishing warehouse $i$ at storage capacity level $l$, retrofitting level $r$, and period $t$.

$E_{n i}$ : Amount of input criterion $n$ for warehouse $i$.

$O_{m i}$ : Amount of output criterion $m$ for warehouse $i$.

$E_{n I D E A L}$ : Amount of input criterion $n$ for virtual ideal warehouse; $E_{n I D E A L}=\min _{i}\left\{E_{n i}\right\}$

$O_{m I D E A L}:$ Amount of output criterion $m$ for virtual ideal warehouse; $O_{m I D E A L}=\max _{i}\left\{O_{n i}\right\}$

\footnotetext{
1. In the DEA problems that represent a form of the production process, input and output criteria are usually the used or required resources and outcomes, respectively. However, in the DEA problems that are considered as a general benchmarking problem, the input and output criteria are usually the-less-the-better type and the-more-the-better type, respectively (Wojcik, Dyckhoff and Clermont, 2019).
} 
$d_{j s}$ : Demand at demand point $j$ under scenario $s$.

$w_{j}$ : Priority of demand point $j$.

$p_{s}$ : Occurrence probability of scenario $s$.

$\rho_{\text {irs }}$ : Percentage of stocked inventory at warehouse $i$ with retrofitting level $r$ that remains usable after the occurrence of scenario $s\left(\rho_{\text {irs }} \leq 1\right)$.

$i r t$ : Interest rate at period $t$.

$B$ : Total budget planned for warehouses establishment.

$B_{t}$ : Part of total budget planned for warehouses establishment that is available at period $t ; \sum_{t \in T} B_{t}=B$.

$A$ : Total budget planned for procuring of relief items.

$A_{t}$ : Part of total budget planned for procuring relief items that is available at period $t ; \sum_{t \in T} A_{t}=A$.

$\theta$ : Percentage of average total demand; $\theta \geq 1$

$\beta_{1}$ : Weight factor in the first objective function.

$\beta_{2}$ : Weight factor in the second objective function.

$t_{i j s}$ : Travel time between warehouse $i$ and demand point $j$ under scenario $s$.

$c_{l}$ : Storage capacity at level $l$.

$F\left(t_{i j s}\right)$ : Deprivation cost function; $F\left(t_{i j s}\right)=0.9814 e^{0.0188 t_{i j s}}$

\section{Decision variables:}

$x_{\text {ilrt }}: 1$, if warehouse $i$ is established at storage capacity level $l$, retrofitting level $r$, and period $t$; 0 , otherwise.

$\vartheta_{n}$ : Weight of input criterion $n$.

$\tau_{m}$ : Weight of output criterion $m$.

$b_{t}$ : The remaining of the establishment budget at the end of period $t$.

$a_{t}$ : The remaining of the procurement budget at the end of period $t$.

$y_{i j s t}$ : Quantity of relief package sent from warehouse $i$ to demand point $j$ under scenario $s$ when the disaster has occurred for the first time at period $t$.

$s_{i t}$ : Quantity of relief package that is bought for warehouse $i$ at period $t$.

\section{3. The Proposed Mathematical Programming Model}

According to the defined symptoms, parameters, and variables, the proposed mathematical programming model comprises three objective functions and fifteen categories of constraints. Following the introduction of the model, more explanations are provided about the objective functions and constraints.

$\operatorname{Min} Z_{1}=\beta_{1}\left(\sum_{i \in I} \sum_{l \in L} \sum_{r \in R} \sum_{t \in T} x_{i l r t}\left\{\sum_{n \in N} \vartheta_{n}\left(E_{n i}-E_{n I D E A L}\right)+\sum_{m \in M} \tau_{m}\left(O_{m I D E A L}-\right.\right.\right.$ $\left.\left.\left.O_{m i}\right)\right\}\right)+\left(1-\beta_{1}\right)\left(\max _{i \in I}\left\{\sum_{l \in L} \sum_{r \in R} \sum_{t \in T} x_{i l r t}\left\{\sum_{n \in N} \vartheta_{n}\left(E_{n i}-E_{n I D E A L}\right)+\sum_{m \in M} \tau_{m}(\right.\right.\right.$

$\left.\left.\left.\left.O_{m I D E A L}-O_{m i}\right)\right\}\right\}\right)$

$\operatorname{Max} Z_{2}=\beta_{2}\left(\sum_{i \in I} \sum_{j \in J ; d_{j s} \neq 0} \sum_{s \in S} \sum_{t \in T} p_{s} w_{j} \frac{\frac{y_{i j s t}}{d_{j s}}}{F\left(t_{i j s}\right)}\right)+\left(1-\beta_{2}\right)\left(\sum_{s \in S} \sum_{t \in T} p_{s} \min _{j \in J ; d_{j s} \neq 0}\{\right.$

$\left.\left.\sum_{i \in I} w_{j} \frac{\frac{y_{i j s t}}{d_{j s}}}{F\left(t_{i j s}\right)}\right\}\right)$

$\operatorname{Min} Z_{3}=\sum_{i \in I} \sum_{l \in L} \sum_{r \in R} \sum_{t \in T} f_{i l r t} x_{i l r t}+\sum_{i \in I} \sum_{t \in T} p c_{i t} s_{i t}$

S. T. : 
$\sum_{l \in L} \sum_{r \in R} \sum_{t \in T} x_{i l r t} \leq 1$

$\sum_{i \in I} \sum_{l \in L} \sum_{r \in R} f_{i l r t} x_{i l r t}+b_{t}=B_{t}+b_{t-1}\left(1+i r_{t-1}\right)$

$\sum_{i \in I} p c_{i t} s_{i t}+a_{t}=A_{t}+a_{t-1}\left(1+i r_{t-1}\right)$

$\sum_{m \in M} \tau_{m} O_{m i} \leq \sum_{n \in N} \vartheta_{n} E_{n i}$

$\sum_{n \in N} \vartheta_{n} E_{n I D E A L}=1$

$\sum_{m \in M} \tau_{m} O_{\text {mIDEAL }}=1$

$\sum_{i \in I} y_{i j s t} \leq d_{j s}$

$\sum_{j \in J} y_{i j s t} \leq \sum_{l \in L} \sum_{r \in R} \sum_{t \in T ; i \leq t} x_{i l r t} \rho_{i r s} s_{i t}$

$\sum_{t \in T} s_{i t} \leq \sum_{l \in L} \sum_{r \in R} \sum_{t \in T} c_{l} x_{i l r t}$

$s_{i t} \leq \sum_{\hat{t} \in T ; \dot{t} \leq t} \sum_{l \in L} \sum_{r \in r} c_{l} x_{i l r t}$

$\sum_{i \in I} \sum_{t \in T} s_{i t} \leq \theta\left(\sum_{j \in J} \sum_{s \in S} p_{s} d_{j s}\right)$

$\tau_{m}, \vartheta_{n} \geq \varepsilon$

$x_{\text {ilrt }} \in\{0,1\}$

$y_{i j s t}, s_{i t} \geq 0$ and Integer

$b_{t}, a_{t} \geq 0$ $\forall i \in I$

$\forall t \in T ; b_{0}=0$

$\forall t \in T ; a_{0}=0$

$\forall i \in I$

$\forall j \in J, s \in S, t \in T$

$\forall i \in I, s \in S, t \in T$

$\forall i \in I$

$\forall i \in I, t \in T$

The first objective function (Eq. (2)) minimizes the weighted sum of the sum of utility scores of the established warehouses and the maximum values of these scores based on the considered criteria. It should be noted that the utility score of each warehouse is calculated based on its weighted coordinate distance from the virtual ideal warehouse; hence the less, the better. In fact, this objective function maximizes warehouses utility. The first expression of the second objective function (Eq. (3)) maximizes fulfilled demands and minimizes deprivation costs or human suffering simultaneously considering the priority of demand points; while the second expression indicates the equitable distribution of commodities according to deprivation cost and the amount of fulfilled demand considering the priority of demand points. The third objective function minimizes the costs of establishing warehouses and procuring relief items. According to Eq. (5), each warehouse can be established in a maximum of one period and a single storage level and retrofitting level. Eq. (6) and Eq. (7) show the constraints of the establishment budget and procurement budget at each period, respectively. Eq. (8) determines that the weighted sum of output criteria is less than or equal to the weighted sum of input criteria. Indeed, this constraint states that the efficiency score of each warehouse (the ratio of the weighted summation of output criteria to the weighted summation of input criteria) is less than or equal to 1. Eq. (9) and Eq. (10) consider both the weighted summation of the input criteria of the virtual ideal warehouse and the weighted summation of the output criteria of the virtual ideal warehouse as equal to 1. Eq. (11) shows that the number of packages sent the demand point cannot exceed its demand. Eq. (12) confirms that dispatched packages from the warehouse cannot exceed the number of packages that remain usable post-disaster. Eq. (13) indicates the maximum storage capacity in the warehouse over the planning time horizon. According to Eq. (14), if the warehouse has not been established up to period $t$, then no goods can be stored in it at period $t$. Eq. (15) represents the maximum storage capacity in the network over the planning time horizon. Eqs. (16) - (19) indicate the type of decision variables applied in the model.

\section{4. Solution Procedure for the Proposed Model}

The programming model presented in the previous section is a stochastic multi-objective mixedinteger non-linear programming model. Therefore, in order to simplify the solution for the model, the following solution is suggested: 


\section{O Linearization of the model}

Since the first and second objective functions and constraint (12) are non-linear, the linear programming model equivalent to the proposed model is presented as follows:

$\operatorname{Min} \dot{Z}_{1}=\beta_{1} \sum_{i \in I} \dot{x}_{i}+\left(1-\beta_{1}\right) \delta$

$\operatorname{Max} \dot{Z}_{2}=\beta_{2}\left(\sum_{i \in I} \sum_{j \in J ; d_{j s} \neq 0} \sum_{s \in S} \sum_{t \in T} p_{s} w_{j} \frac{\frac{y_{i j s t}}{d_{j s}}}{F\left(t_{i j s}\right)}\right)+\left(1-\beta_{2}\right)\left(\sum_{s \in S} \sum_{t \in T} p_{s} \sigma_{s t}\right)$

$\operatorname{Min} Z_{3}=\sum_{i \in I} \sum_{l \in L} \sum_{r \in R} \sum_{t \in T} f_{i l r t} x_{i l r t}+\sum_{i \in I} \sum_{t \in T} p c_{i t} s_{i t}$

S. T. :

Eqs. (5) - (11) and (13) - (19)

$\dot{x}_{i} \leq G\left(\sum_{l \in L} \sum_{r \in R} \sum_{t \in T} x_{i l r t}\right) \quad \forall i \in I$

(22)

$\dot{x}_{i} \leq \sum_{n \in N} \vartheta_{n}\left(E_{n i}-E_{n I D E A L}\right)+\sum_{m \in M} \tau_{m}\left(O_{m I D E A L}-O_{m i}\right) \quad \forall i \in I$

$\dot{x}_{i} \geq \sum_{n \in N} \vartheta_{n}\left(E_{n i}-E_{n I D E A L}\right)+\sum_{m \in M} \tau_{m}\left(O_{m I D E A L}-O_{m i}\right)+\forall i \in I$

$G\left(\sum_{l \in L} \sum_{r \in R} \sum_{t \in T} x_{i l r t}-1\right)$

$\sum_{i \in I} w_{j} \frac{\frac{y_{i j s t}}{d_{j s}}}{F\left(t_{i j s}\right)} \geq \sigma_{s t}$

$\forall j \in J ; d_{j s} \neq 0, s \in S, t \in T$

$\dot{x}_{i} \leq \delta$

$\forall i \in I$

$\sum_{j \in J} y_{i j s t} \leq \sum_{\hat{t} \in T ; \hat{t} \leq t} \rho_{i r s} s_{i \hat{t}}+G\left(1-\sum_{l \in L} \sum_{\hat{t} \in T ; \dot{t} \leq t} x_{i l r \hat{t}}\right)$

$\forall i \in I, r \in R, s \in S, t \in T(27)$

$\sum_{j \in J} y_{i j s t} \leq \sum_{\hat{t} \in T ; \hat{t} \leq t} S_{i \hat{t}}$

$\forall i \in I, s \in S, t \in T$

$\dot{x}_{i}, \delta, \sigma_{s t} \geq 0$

$\forall i \in I, s \in S, t \in T$

$G, G$ are a large enough positive constant.

\section{o Transforming the multi-objective model into a single-objective model}

In this study, the model is transformed into a single-objective model using the fuzzy multiobjective programming model presented by Lin (2004) (Weighted Max-Min model):

Step 1: Calculating the minimum and maximum values of each objective function

Generally, the multi-objective programming model can be defined as follows:

$\operatorname{Min} Z_{1}, Z_{2}, \ldots, Z_{n}$

$\operatorname{Max} W_{1}, W_{2}, \ldots, W_{m}$

S. T.:

$x \in X$

The minimum value of each objective function $\left(W_{j}^{-}, Z_{i}^{-}\right)$and the maximum value of each objective function $\left(W_{j}^{+}, Z_{i}^{+}\right)$can be obtained as follows:
$=\operatorname{Max} Z_{i} ;$ S. T.: $\mathrm{x} \in X Z_{i}^{+}$
$=\operatorname{Min} Z_{i} ;$ S. T.: $\mathrm{x} \in X Z_{i}^{-}$
(33) $\forall i \in\{1,2, \ldots, n\}$
$=\operatorname{Max} W_{j} ;$ S. T.: $x \in X W_{j}^{+}$
(34) $\forall i \in\{1,2, \ldots, n\}$
$=\operatorname{Min} W_{j} ;$ S. T.: $x \in X W_{j}^{-}$
(35) $\forall j \in\{1,2, \ldots, m\}$
$(36) \forall j \in\{1,2, \ldots, m\}$ 


\section{Step 2: Defining the membership function of each objective function}

The values of the objective functions can be shown as fuzzy numbers; accordingly, the value of their membership function changes linearly between zero and one. Such linear membership functions are defined below and illustrated in Fig. 7.

$$
\begin{aligned}
\mu_{Z_{i}} & = \begin{cases}1 & Z_{i}^{-} \leq Z_{i} \\
\left(Z_{i}^{+}-Z_{i}\right) /\left(Z_{i}^{+}-Z_{i}^{-}\right) & Z_{i}^{-} \leq Z_{i} \leq Z_{i}^{+} \\
0 & Z_{i}^{+} \geq Z_{i}\end{cases} \\
\mu_{W_{j}} & =\left\{\begin{array}{ll}
1 & W_{j}^{+} \leq W_{j} \\
\left(W_{j}-W_{j}^{-}\right) /\left(W_{j}^{+}-W_{j}^{-}\right) & W_{j}^{-} \leq W_{j} \leq W_{j}^{+} \\
0 & W_{j}^{-} \leq W_{j}
\end{array} \quad(38) \forall i \in\{1,2, \ldots, n\} \in\{1,2, \ldots, m\}\right.
\end{aligned}
$$

\section{Step 3: Formulating a corresponding single-objective programming model}

The above multi-objective programming model is transferred into a single-objective programming model below, with the aim of maximizing the minimum membership functions of the objective functions and taking the weight of the objective functions into account.

$\operatorname{Max} \bar{Z}=\lambda$

S. T.:

$$
\begin{array}{ll}
\alpha_{i} \lambda \leq \mu_{Z_{i}} & (40) \forall i \in\{1,2, \ldots, n\} \\
\alpha_{j} \lambda \leq \mu_{W_{j}} & \forall j \in\{1,2, \ldots, m\} \\
x \in X &
\end{array}
$$

Where $\alpha_{i}, \alpha_{j}$ represent the weights of the objective functions.

Next, according to the weighted Max-Min model and the proposed linearization approach, the proposed programming model is transferred into the below single-objective linear programming model.
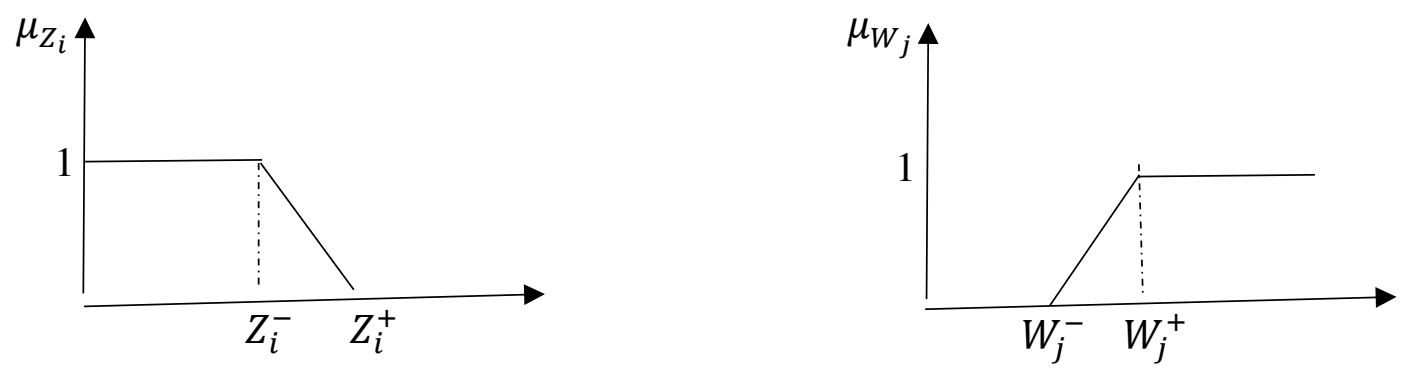

Fig. 7. Membership function for objective functions. 
$\operatorname{Max} \bar{Z}=\lambda$

S. T.:

$\alpha_{1} \lambda \leq\left(Z_{1}^{+}-\dot{Z}_{1}\right) /\left(Z_{1}^{+}-Z_{1}^{-}\right)$

$\alpha_{2} \lambda \leq\left(\dot{Z}_{2}-\dot{Z}_{2}^{-}\right) /\left(\dot{Z}_{2}^{+}-\dot{Z}_{2}^{-}\right)$

$\alpha_{3} \lambda \leq\left(Z_{3}^{+}-Z_{3}\right) /\left(Z_{3}^{+}-Z_{3}^{-}\right)$

Eqs. (5) - (11), (13) - (19), and (22) - (29)

It is worth noting that due to the special nature of the first objective function and the model structure, the maximum value of the first objective function $\left(Z_{1}^{+}\right)$becomes an unlimited value by solving the model and maximizing only the first objective function. Therefore, to obtain a finite value for $Z_{1}^{+}$, the model is solved by maximizing only the first objective function and substituting Eq. 26 with the following equations.

$$
\begin{array}{lc}
\delta \leq \dot{x}_{i}+G * O_{i} & \forall i \in I \\
\sum_{i \in I} O_{i}=|I|-1 & \forall i \in I \\
O_{i} \in\{0,1\} & \forall i \in
\end{array}
$$

\section{Numerical Example}

In this example, the occurrence of an earthquake in an area is taken into account. Its data were randomly estimated based on their sample values in studies similar to real conditions. Furthermore, the following hypotheses were considered in this example:

- Five demand points and ten candidate sites for establishing warehouses were considered.

- The planning time horizon was divided into three periods.

$\circ$ Four disaster scenarios with the probabilities of occurrence of $0.45,0.3,0.1$, and 0.15 were considered.

- Warehouses can be established in three sizes (small, medium, and large) and at two retrofitting levels (medium, and high). Information on establishment cost and storage capacity of each size are presented in Table 2. Besides, at the medium retrofitting level, the percentage of stored packages that remain usable post-disaster is estimated as shown in Table 3. It is worth mentioning that at the high retrofitting level, $100 \%$ of relief packages are usable.

- Relief items packaged in packages included drinking water and food with long expiration dates and shelf life. Food was stocked in the form of meals-ready-to-eat (MREs). MRE is a type of individual operational ration that will simply be referred to as ration. The ration includes a variety of food items for breakfast, lunch, and dinner and an MRE provides about 1.3 of the human body's daily need for calories. A person's daily need for water and food is three liters and two MREs, respectively. Here, three 24-hour periods were considered to meet demands. Subsequently, each package contains 9 liters of water and 6 MREs. The costs of procuring each package for each warehouse are shown in Table 4. 
- Input criteria: ease of access to transport networks ${ }^{1}\left(I_{1}\right)$, ease of access to supply resources of relief goods ${ }^{2}$ (i.e. suppliers) $\left(I_{2}\right)$, and difficulty of traversing on routes leading to the warehouse $\left(I_{3}\right)$ and output criteria: security of warehouse and its routes $\left(O_{1}\right)$, geographical and climatic conditions $\left(\mathrm{O}_{2}\right)$, ease of access to essential infrastructure such as water, electricity, gas, etc. $\left(\mathrm{O}_{3}\right)$, and quality of supply resources of relief goods (based on production capacity, flexibility, delivery time, disruption risk, economic stability, etc.) $\left(\mathrm{O}_{4}\right)$ were considered. Also, the scores obtained from the evaluation of warehouses based on the mentioned criteria were randomly generated in the range of $[1,0]$, which are presented in Table 5.

○ Travel times, demand values ${ }^{3}$, the priority of the demand points, and the rest of the information are presented in Tables 6-8, respectively.

Table 2. Cost of establishing warehouses at each storage and retrofitting level, and period $\left(10^{6}\right.$ Tomans).

\begin{tabular}{c|ll|cc|cc}
\hline Storage level & \multicolumn{2}{|c|}{ Small (30000 packages) } & \multicolumn{2}{c}{ Medium $\mathbf{( 4 8 0 0 0}$ packages) } & \multicolumn{2}{c}{ Large (72000 packages) } \\
\hline Retrofitting level & Medium & High & Medium & High & Medium & High \\
\hline Warehouse 1 & $(118,122,126)$ & $(138,142,147)$ & $(189,195,201)$ & $(221,228,236)$ & $(283,291,301)$ & $(331,341,352)$ \\
\hline Warehouse 2 & $(94,102,111)$ & $(121,131,142)$ & $(150,162,176)$ & $(194,210,228)$ & $(226,244,265)$ & $(290,313,340)$ \\
\hline Warehouse 3 & $(128,131,134)$ & $(167,170,174)$ & $(205,209,214)$ & $(267,272,279)$ & $(307,313,321)$ & $(401,409,419)$ \\
\hline Warehouse 4 & $(149,151,154)$ & $(196,199,203)$ & $(238,242,247)$ & $(314,319,325)$ & $(358,363,370)$ & $(470,477,487)$ \\
\hline Warehouse 5 & $(92,100,110)$ & $(115,125,138)$ & $(147,160,176)$ & $(184,201,221)$ & $(221,241,265)$ & $(276,301,331)$ \\
\hline Warehouse 6 & $(140,147,156)$ & $(182,191,202)$ & $(224,235,249)$ & $(291,306,324)$ & $(336,353,374)$ & $(437,459,487)$ \\
\hline Warehouse 7 & $(125,129,134)$ & $(170,176,183)$ & $(200,207,215)$ & $(272,282,293)$ & $(300,311,323)$ & $(408,422,438)$ \\
\hline Warehouse 8 & $(134,135,137)$ & $(166,168,171)$ & $(214,216,219)$ & $(266,269,273)$ & $(322,325,330)$ & $(398,402,408)$ \\
\hline Warehouse 9 & $(119,122,126)$ & $(144,147,151)$ & $(190,195,201)$ & $(230,236,243)$ & $(286,293,301)$ & $(346,354,364)$ \\
\hline Warehouse 10 & $(99,109,121)$ & $(142,156,173)$ & $(158,174,193)$ & $(227,250,278)$ & $(238,262,291)$ & $(341,375,416)$ \\
\hline
\end{tabular}

- Numbers inside parentheses in each cell from left to right represent the expense of establishing warehouses at the first, second, and third periods, respectively.

Table 3. Percentage of stocked packages that remain usable post-disaster.

\begin{tabular}{ccccc}
\hline & Scenario 1 & Scenario 2 & Scenario 3 & Scenario 4 \\
\hline Warehouse 1 & $\% 100$ & $\% 87$ & $\% 91$ & $\% 95$ \\
\hline Warehouse 2 & $\% 90$ & $\% 100$ & $\% 92$ & $\% 77$ \\
\hline Warehouse 3 & $\% 90$ & $\% 100$ & $\% 77$ & $\% 87$ \\
\hline Warehouse 4 & $\% 85$ & $\% 100$ & $\% 75$ & $\% 100$ \\
\hline Warehouse 5 & $\% 100$ & $\% 75$ & $\% 94$ & $\% 87$ \\
\hline Warehouse 6 & $\% 80$ & $\% 90$ & $\% 100$ & $\% 100$ \\
\hline Warehouse 7 & $\% 95$ & $\% 100$ & $\% 96$ & $\% 100$ \\
\hline Warehouse 8 & $\% 92$ & $\% 88$ & $\% 100$ & $\% 78$ \\
\hline Warehouse 9 & $\% 90$ & $\% 100$ & $\% 81$ & $\% 100$ \\
\hline Warehouse 10 & $\% 90$ & $\% 100$ & $\% 100$ & $\% 100$ \\
\hline
\end{tabular}

\footnotetext{
1. This criterion is calculated as the weighted sum of the ease of access to various types of transportation networks such as airbases, airports, train stations, cargo stations, etc. Ease of access is also assessed based on the condition and the distance of the route between the warehouse and the transportation network.

${ }^{2}$. Ease of access is measured based on the condition and the distance of the route between the warehouse and the supply resource.

3. The extent of the demand in the affected area can be estimated based on its population and the severity of the inflicted damage. Also, the severity of the inflicted damage can be calculated according to the type of soil, urban texture and buildings, the condition of arterial roads, and the slope of the land in the affected area. Moreover, the priority of demand points can be determined based on various criteria such as distance from causal faults, landslide, structural behavior of buildings and infrastructure, electricity, gas, water and sewage network, transportation network, medical emergency services, fire stations, demographic characteristics (age, gender, income level, health status), rescue capability, and mortality ratio.
} 
Table 4. Cost of procuring a package $\left(10^{4}\right.$ Tomans $)$.

\begin{tabular}{cccc}
\hline & period 1 & period 2 & period 3 \\
\hline Warehouse 1 & 2 & 4 & 7 \\
\hline Warehouse 2 & 5 & 6 & 8 \\
\hline Warehouse 3 & 4 & 6 & 7 \\
\hline Warehouse 4 & 3.5 & 5 & 6.5 \\
\hline Warehouse 5 & 3 & 6 & 8 \\
\hline Warehouse 6 & 2.5 & 3 & 5 \\
\hline Warehouse 7 & 1 & 3 & 5 \\
\hline Warehouse 8 & 4.5 & 6 & 9 \\
\hline Warehouse 9 & 5.5 & 6.5 & 8 \\
\hline Warehouse 10 & 2 & 3 & 6 \\
\hline
\end{tabular}

Table 5. Scores obtained from the evaluation of warehouses based on the input and output criteria.

\begin{tabular}{cccccccc}
\hline & $\boldsymbol{I}_{\mathbf{1}}$ & $\boldsymbol{I}_{\mathbf{2}}$ & $\boldsymbol{I}_{\mathbf{3}}$ & $\boldsymbol{O}_{\mathbf{1}}$ & $\boldsymbol{O}_{\mathbf{2}}$ & $\boldsymbol{O}_{\mathbf{3}}$ & $\boldsymbol{O}_{4}$ \\
\hline Warehouse 1 & 0.6 & 0.4 & 0.9 & 0.4 & 1 & 0.5 & 0.14 \\
\hline Warehouse 2 & 0.8 & 0.1 & 0.1 & 0.2 & 0.7 & 0.13 & 0.1 \\
\hline Warehouse 3 & 0.3 & 0.8 & 0.14 & 0.7 & 0.8 & 0.9 & 0.7 \\
\hline Warehouse 4 & 0.1 & 0.15 & 0.2 & 0.17 & 0.17 & 0.16 & 0.15 \\
\hline Warehouse 5 & 0.5 & 0.11 & 0.1 & 0.14 & 0.15 & 1 & 0.3 \\
\hline Warehouse 6 & 0.17 & 0.15 & 0.15 & 0.17 & 0.1 & 0.17 & 0.5 \\
\hline Warehouse 7 & 0.14 & 0.3 & 0.17 & 0.1 & 0.5 & 0.5 & 0.12 \\
\hline Warehouse 8 & 0.4 & 0.2 & 0.1 & 0.15 & 0.17 & 0.3 & 0.8 \\
\hline Warehouse 9 & 0.5 & 0.17 & 0.6 & 0.2 & 0.17 & 0.8 & 0.7 \\
\hline Warehouse 10 & 0.21 & 0.1 & 0.8 & 0.4 & 0.3 & 0.16 & 0.11 \\
\hline Virtual ideal warehouse & 0.1 & 0.1 & 0.1 & 0.7 & 1 & 1 & 0.8 \\
\hline
\end{tabular}

Table 6. Travel times under each scenario (In hours).

\begin{tabular}{lccccc}
\hline & Demand point 1 & Demand point 2 & Demand point 3 & Demand point 4 & Demand point 5 \\
\hline Warehouse 1 & $(0.7,0.5,0.9,0.6)$ & $(2.5,2.3,1.9,1.8)$ & $(3.2,3.9,3,2.9)$ & $(2.65,2.1,2.3,2.9)$ & $(3.5,3.3,3.1,2.9)$ \\
\hline Warehouse 2 & $(0.65,0.45,0.85,0.55)$ & $(2.4,2.2,1.8,1.7)$ & $(3.1,3.8,2.9,2.8)$ & $(2.55,2,2.2,2.8)$ & $(3.4,3.2,3,2.8)$ \\
\hline Warehouse 3 & $(0.7,0.6,0.8,0.75)$ & $(2,1.7,1.3,1.5)$ & $(2.9,2.4,3.2,2.6)$ & $(1.8,1.6,2,1.5)$ & $(2.8,2.4,3,2.6)$ \\
\hline Warehouse 4 & $(0.5,0.3,0.6,0.4)$ & $(2.4,2.2,2.8,2.5)$ & $(3.7,3.2,4,3.5)$ & $(2.6,2.3,2.8,2.4)$ & $(3.5,3.1,3.7,3.3)$ \\
\hline Warehouse 5 & $(1.9,1.7,1.5,1.4)$ & $(0.3,0.4,0.25,0.25)$ & $(1.7,1.5,1.4,1.2)$ & $(2.3,2.1,2,1.8)$ & $(1.9,1.7 .1 .6,1.4)$ \\
\hline Warehouse 6 & $(2.8,3,2.5,2.6)$ & $(1.5,1.8,1.25,1.35)$ & $(0.4,0.5,0.3,0.3)$ & $(3.1,3.5,2.9,2.8)$ & $(2,2.3,1.8,1.8)$ \\
\hline Warehouse 7 & $(1.8,1.6,1.9,2)$ & $(2.2,1.8,2,2.4)$ & $(3.5,3,3.2,3.6)$ & $(0.3,0.2,0.25,0.35)$ & $(1.1,0.9,1,1.2)$ \\
\hline Warehouse 8 & $(3,2.75,2.5,2.6)$ & $(2,1.7,1.5,1.55)$ & $(2.2,2,1.8,1.85)$ & $(1.2,1,0.85,0.9)$ & $(0.3,0.2,0.15,0.15)$ \\
\hline Warehouse 9 & $(1.6,1.9,1.7,1.45)$ & $(2,2.4,2.2,1.9)$ & $(1.8,2.1,1.9,1.65)$ & $(3,3.5,3.2,2.9)$ & $(4,4.3,4.15,3.85)$ \\
\hline Warehouse 10 & $(2.2,1.8,2.5,2.7)$ & $(2.8,2.6,2.4,2.7)$ & $(3.5,3.9,3.6,4)$ & $(1.9,1.7,2.1,2.5)$ & $(2.9,2.7,2.7,3)$ \\
\hline
\end{tabular}

Table 7. Demand values under each scenario $\left(10^{3}\right.$ packages $)$ along with the priority of the demand points.

\begin{tabular}{lccccc}
\hline & $\boldsymbol{W}_{\boldsymbol{j}}$ & Scenario 1 & Scenario 2 & Scenario 3 & Scenario 4 \\
\hline Demand point 1 & 0.25 & 0 & 70 & 100 & 0 \\
\hline Demand point 2 & 0.3 & 45 & 90 & 0 & 80 \\
\hline Demand point 3 & 0.2 & 65 & 0 & 40 & 100 \\
\hline Demand point 4 & 0.1 & 90 & 80 & 110 & 70 \\
\hline Demand point 5 & 0.15 & 80 & 60 & 0 & 100 \\
\hline
\end{tabular}

Table 8. The rest of the required information.

\begin{tabular}{|c|c|c|c|c|c|c|c|}
\hline & period 1 & period 2 & period 3 & $\alpha_{1}$ & 0.3 & $\boldsymbol{\beta}_{2}$ & 0.6 \\
\hline Establishment budget $\left(1^{6}{ }^{6}\right.$ Tomans $)\left(B_{t}\right)$ & 400 & 500 & 600 & $\alpha_{2}$ & 0.6 & $\theta$ & 1.2 \\
\hline Procurement budget $\left(1^{6}{ }^{6}\right.$ Tomans) $\left(A_{t}\right)$ & 300 & 400 & 550 & $\alpha_{3}$ & 0.1 & $G$ & $10^{3}$ \\
\hline Interest rate $\left(i r_{t}\right)$ & $\% 10$ & $\% 12$ & $\% 15$ & $\beta_{1}$ & 0.6 & $\dot{G}$ & $10^{6}$ \\
\hline
\end{tabular}


The proposed programming model was solved in IBM ILOG CPLEX software. The obtained results are presented in Table $9-11$ and Figs. $8-10$.

Table 9. Minimum and maximum values of each objective function.

\begin{tabular}{cccccc}
\hline $\boldsymbol{Z}_{\mathbf{1}}^{-}$ & $\boldsymbol{Z}_{\mathbf{1}}^{+}$ & $\boldsymbol{Z}_{\mathbf{2}}^{-}$ & $\boldsymbol{Z}_{2}^{+}$ & $\boldsymbol{Z}_{\mathbf{3}}^{-}$ & $\boldsymbol{Z}_{3}^{+}$ \\
\hline 0 & 22.881 & 0 & 1.12 & 0 & $3.019 \mathrm{E}+9$ \\
\hline
\end{tabular}

Table 10. Selected warehouses along with their specifications, utility scores, and the amount of storage of relief items within them.

\begin{tabular}{cccccccc}
\hline Warehouse & $\begin{array}{c}\text { Establishment } \\
\text { time }\end{array}$ & Storage level & Retrofitting & Utility & \multicolumn{3}{c}{ Stocked packages } \\
\cline { 6 - 8 } & & level & score & period 1 & period 2 & period 3 \\
\hline 2 & 2 & Small & Medium & 0.8725 & 0 & 29866 & 134 \\
\hline 3 & 3 & Medium & Medium & 6.989 & 0 & 0 & 48000 \\
\hline 4 & 3 & Small & High & 1.331 & 0 & 0 & 21423 \\
\hline 5 & 1 & Medium & Medium & 0.329 & 48000 & 0 & 0 \\
\hline 6 & 2 & Small & High & 1.2975 & 0 & 30000 & 0 \\
\hline 7 & 2 & Medium & Medium & 2.524 & 0 & 48000 & 0 \\
\hline 10 & 1 & Large & Medium & 0.88 & 72000 & 0 & 0 \\
\hline
\end{tabular}

Table 11. The rest of the obtained results.

\begin{tabular}{ccc|ccccc}
\hline$\lambda$ & $\boldsymbol{Z}_{\mathbf{1}}^{*}$ & $\boldsymbol{Z}_{\mathbf{2}}^{*}$ & $\boldsymbol{Z}_{\mathbf{3}}^{*}$ & $=$ & $\begin{array}{c}\text { Total cost of establishing } \\
\text { warehouses (Tomans) }\end{array}$ & + & $\begin{array}{c}\text { Total cost of procuring } \\
\text { relief items (Tomans) }\end{array}$ \\
\hline 1.6647 & 11.454 & 1.1187 & $2.4795 \mathrm{E}+9$ & $=$ & $1.302 \mathrm{E}+9$ & + & $1.1775 \mathrm{E}+9$ \\
\hline
\end{tabular}

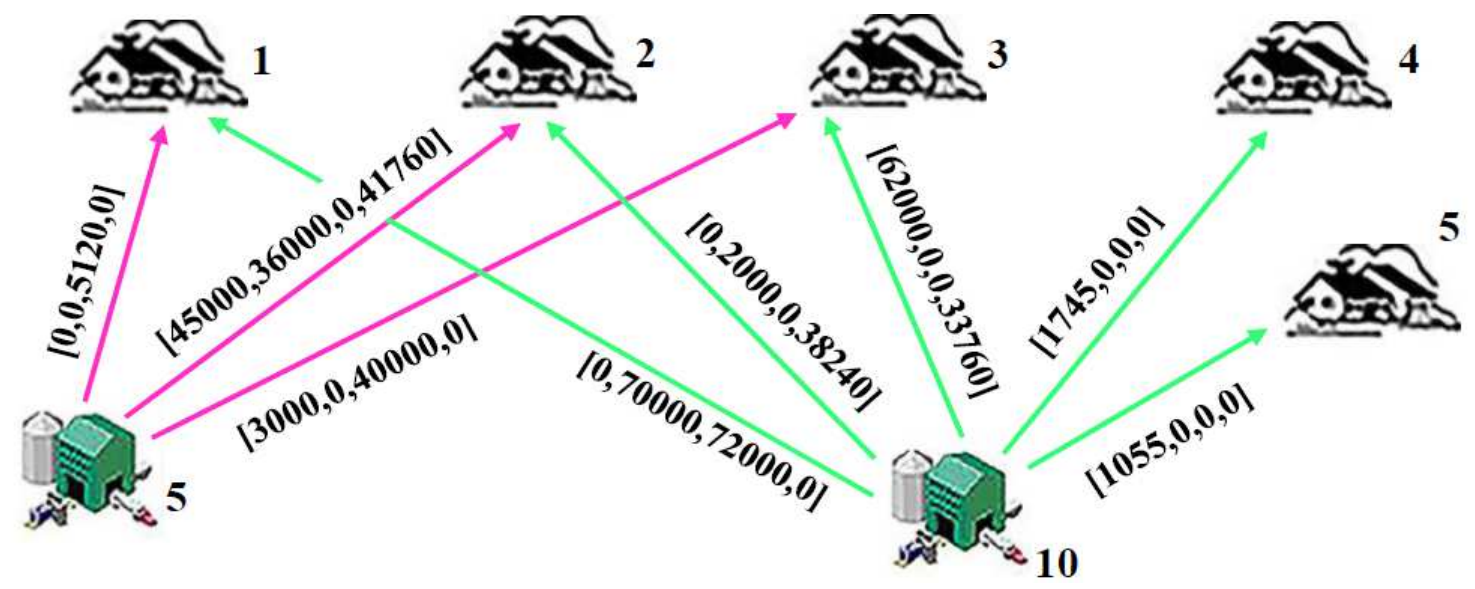

Fig. 8. How relief packages are distributed among demand points at period 1 under each scenario.

- On each arc of the network, numbers inside bracket from left to right represent the number of dispatched packages under the first, second, third, and fourth scenarios, respectively. 


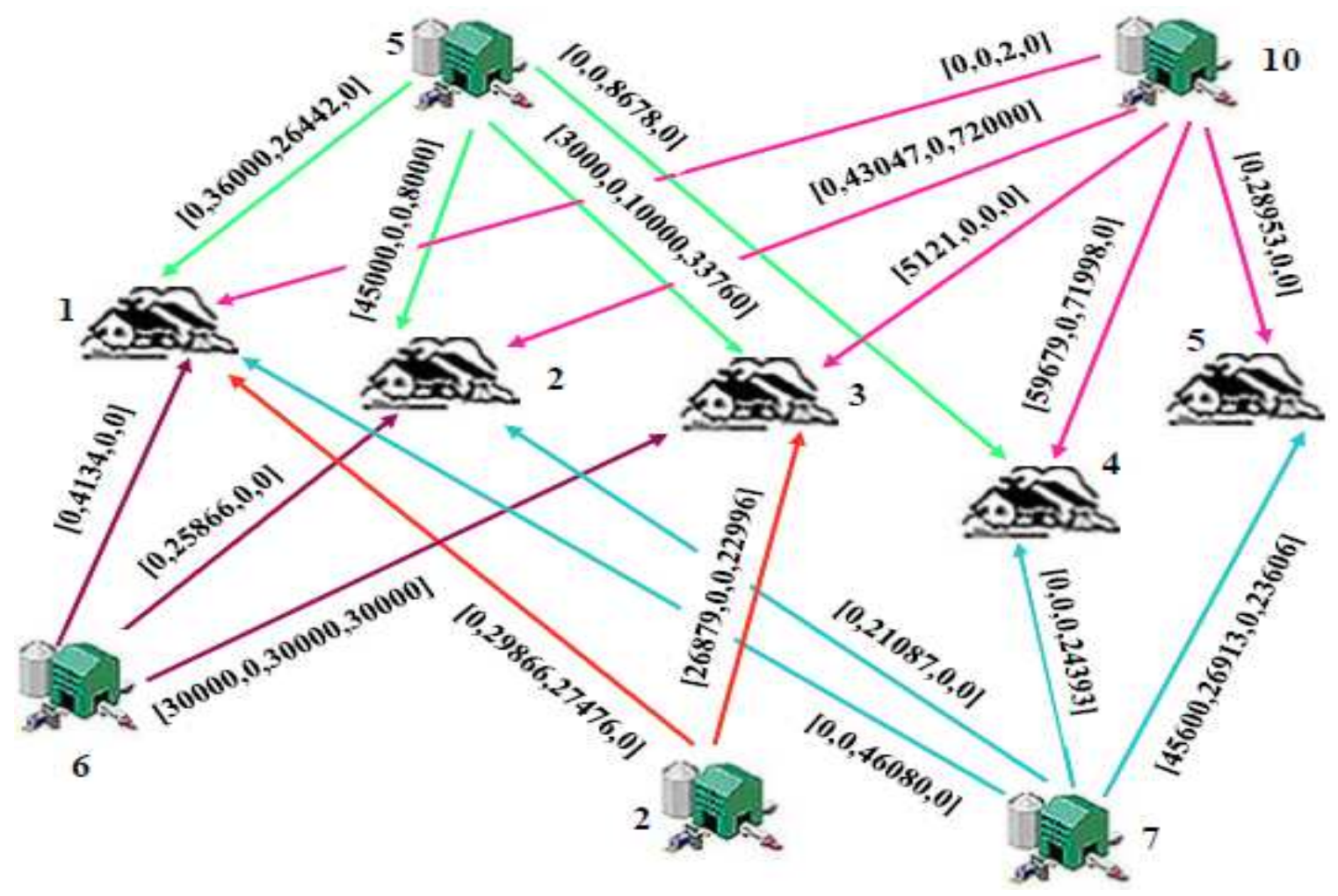

Fig. 9. How relief packages are distributed among demand points at period 2 under each scenario.

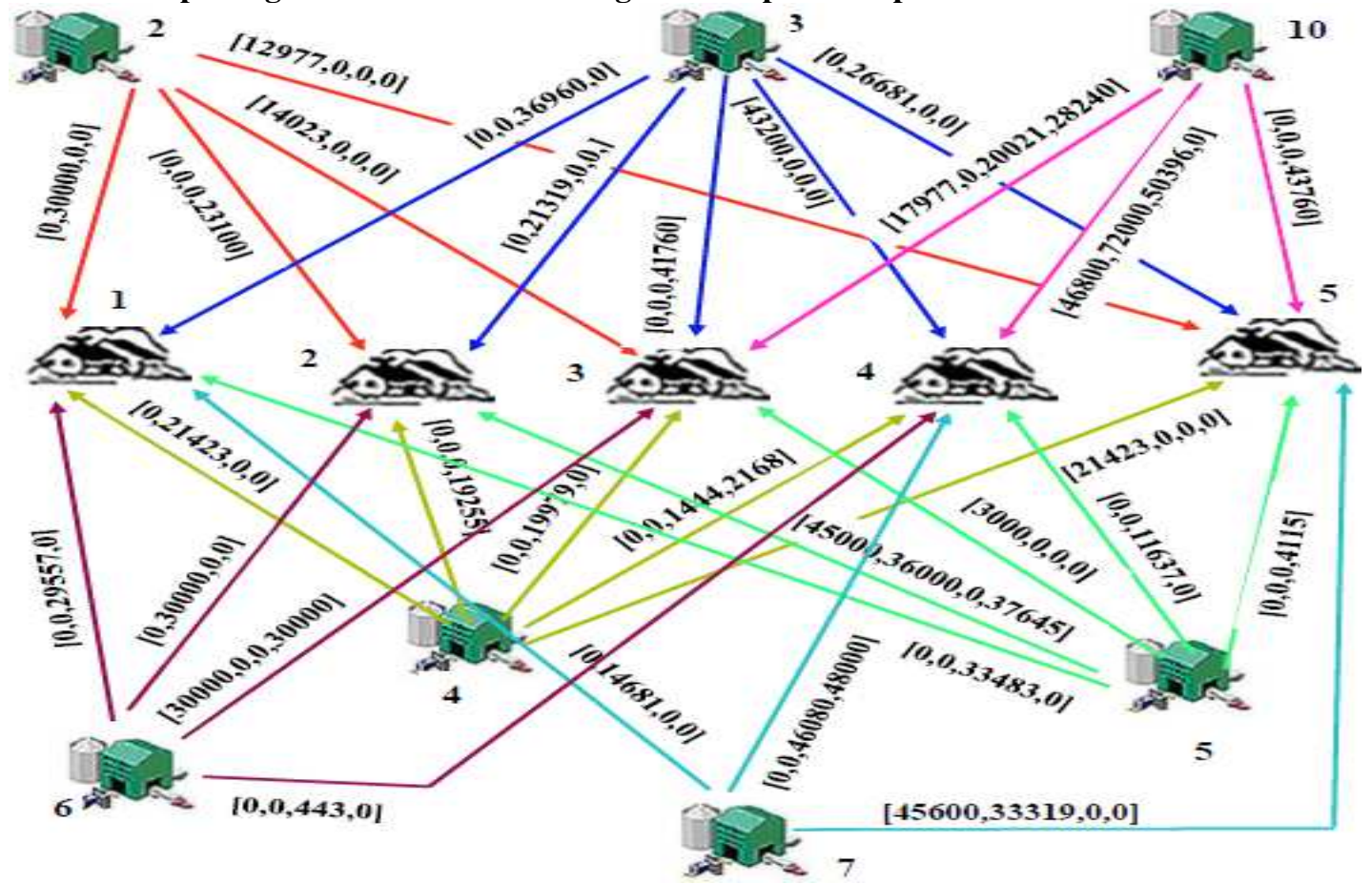

Fig. 10. How relief packages are distributed among demand points at period 3 under each scenario. 


\subsection{Evaluating the Performance Efficiency of the Proposed Programming Model}

In this section, the effects of the formulated hypotheses and adopted approaches on the performance of the model are examined. These evaluations are as follows.

\subsubsection{Assessing the Performance Efficiency of the Proposed Procedure to Establishing the} Most Desirable Warehouses according to a Set of Criteria

In this section, the following three evaluations were carried out to measure the performance efficiency of the proposed approach in line with establishing the most desirable warehouses based on a set of criteria. The results of these evaluations are shown in Fig. 11.

1.1. Evaluating the performance efficiency of the proposed DEA model: The performance efficiency of the proposed DEA model was evaluated by comparing it with the DEA models used in studies done by Klimberg and Ratick (2008) and Afsharian (2019) by maximizing the utility of warehouses based on these DEA models (The proposed model based on each DEA model used in these two studies are provided in the appendix).

1.2. Evaluating the effect of integrating the proposed DEA model with the proposed mathematical programming model: It is assumed that the proposed DEA model (Its formulation is provided in the appendix) is not integrated with the proposed mathematical programming model. In this case, first the efficiency score of each warehouse is calculated using the proposed DEA model and then these scores are considered as an input parameter for the model. Consequently, the first objective function (Eq. 2) is reformulated as follows:

$\operatorname{Max} Z_{l}=\sum_{i \in I} \sum_{l \in L} \sum_{r \in R} \sum_{t \in T} E S_{i} x_{i l r t}$

Where $E S_{i}$ is the efficiency score of warehouse $i$, the calculation method of which is provided in the appendix.

1.3. Evaluating the performance of the second expression of the first objective function (the maximum utility scores of established warehouses based on a set of criteria): As mentioned earlier, the proposed DEA model was formulated by taking inspiration from the DEA model presented by Sun et al. (2013). Yet unlike the model by Sun et al., in addition to minimizing the summation of the weighted coordinate distances between the virtual ideal warehouse and all established warehouses, the proposed DEA model also minimizes the maximum weighted coordinate distances between the virtual ideal warehouse and all established warehouses. In this regard, this expression was omitted from Eq. 2 to assess the performance of the second expression of the first objective function.

In the proposed approach, the second objective function, which has a higher priority for the relief organization, has been realized to a higher extent (Note that the figures presented in Fig. 11 are not considerably different in appearance; however, the observed changes are significant due to the small scale of the second objective function). It is worth noting that the higher the amount of the second objective function, the more satisfied the demands and/or the lower the deprivation costs (i.e. less human suffering and higher response speed). As a result, the proposed approach had a better performance in responding to the affected people. Besides, the proposed approach made the total cost more affordable, given the lower amount of its third objective function. Subsequently, it can be stated that the proposed approach to establishing the most desirable warehouses based on the input and output criteria has a better performance. 


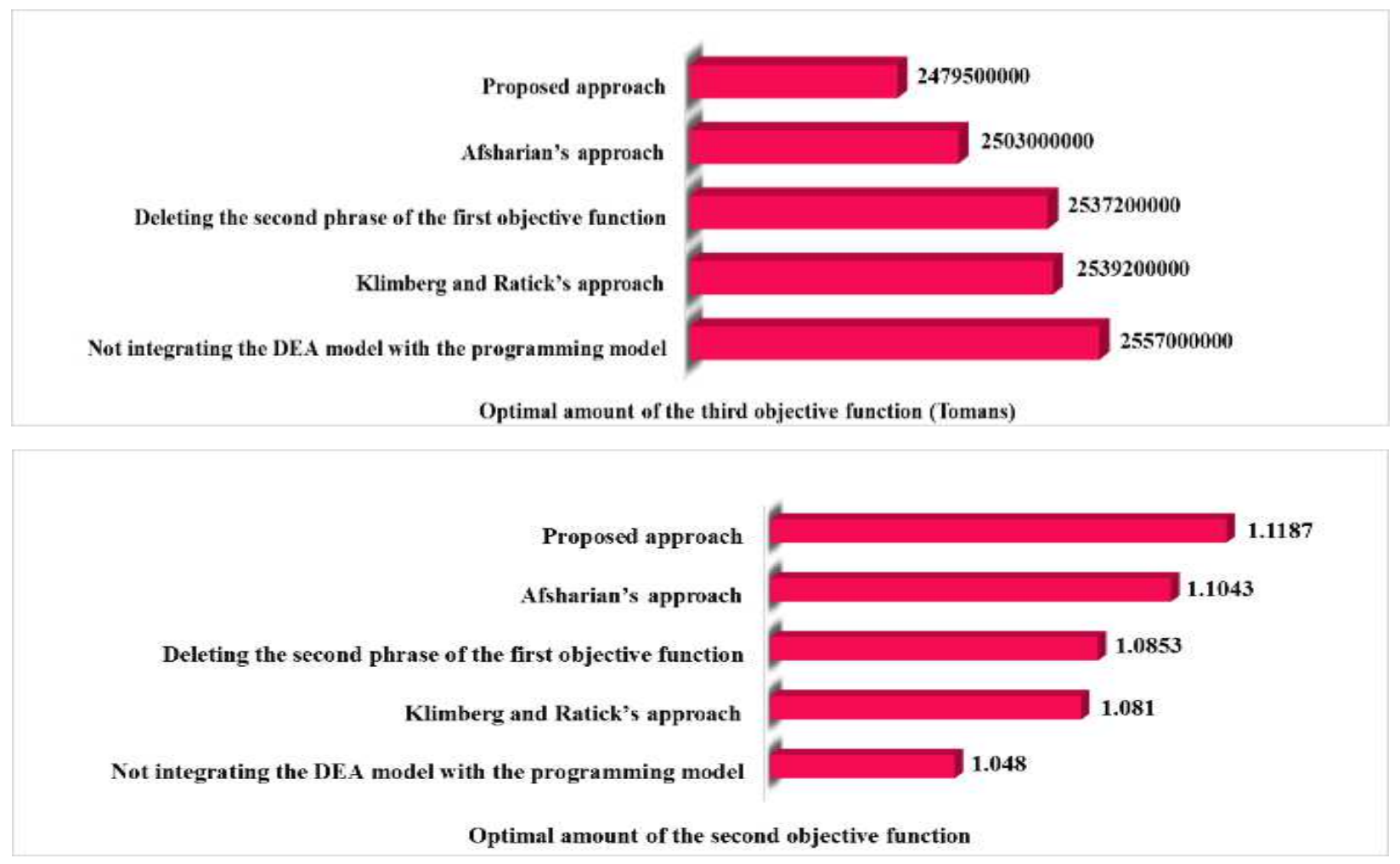

Fig. 11. The results obtained from assessing the performance of the proposed procedure to establishing the most desirable warehouses according to a set of criteria.

\subsubsection{Investigating the Effects of Some of the Considered Hypotheses}

In this section, the following evaluations were carried out to investigate the effects of some of the important and basic hypotheses considered in the problem under discussion.

2.1. Evaluating the performance of deprivation cost function: The deprivation cost function considered in this study was compared with some other deprivation cost functions presented in the literature (Holguín-Veritas et al., 2016); the outcomes are shown in Table 12.

Regarding the results shown in Table 12, it can be stated that the deprivation cost function used in this study outperforms other functions due to the following reasons:

○ It increased the total average satisfied demands; subsequently, it performed better in responding to the affected people.

o Its total cost is more affordable.

- Compared to other functions, it established the same number of warehouses or more, while the amount of the first objective function was lower. Therefore, more desirable warehouses were established based on the intended output and input criteria using this function.

Table 12. Results obtained by comparing the deprivation cost function used in this study with some other functions.

\begin{tabular}{lcccc}
\hline \multicolumn{1}{c}{ Functions } & $\mathbf{0 . 9 8 1 4 \boldsymbol { e } ^ { \mathbf { 0 . 0 1 8 8 } }}$ & $\mathbf{0 . 2 8 6 9 \boldsymbol { e } ^ { \mathbf { 0 . 0 9 9 8 } }}$ & $\mathbf{0 . 2 3 5 4 \boldsymbol { e } ^ { \mathbf { 0 . 1 1 2 9 } }}$ & $\mathbf{0 . 1 9 3 2 \boldsymbol { e } ^ { \mathbf { 0 . 1 2 5 9 } }}$ \\
\hline Optimal amount of the first objective function & 11.454 & 11.818 & 11.865 & 11.789 \\
\hline Optimal amount of the third objective function (Tomans) & $2.4795 \mathrm{E}+9$ & $2.5329 \mathrm{E}+9$ & $2.535 \mathrm{E}+9$ & $2.5316 \mathrm{E}+9$ \\
\hline Total average satisfied demands ${ }^{\mathbf{1}}$ (Packages) & 606190 & 583510 & 567770 & 581540 \\
\hline
\end{tabular}

1. This criterion is calculated as follows: $\sum_{s \in S} \sum_{t \in T} p_{s} y_{i j s t}$ 
2.2. Investigating the effects of taking bank interest into account: In this study, the time value of money was considered in the form of bank interest allocated to the remaining establishment and procurement budgets at the end of a period. Fig. 12 shows the effects of taking into account the time value of money in the proposed programming model by eliminating the interest rate. Taking bank interest into account led to a greater realization of the second objective function which resulted in better performance in responding to affected people. Albeit, bank interest did increase the total cost. Finally, it is noteworthy that the same number of warehouses were established in both; yet, the first objective function involved a more desirable value by considering bank interest. Therefore, taking bank interest into account led to the establishment of more desirable warehouses based on the intended output and input criteria. For the relief organization, the total cost has a lower priority than the other two objective functions. Therefore, as expected, taking the assumed time value into account leads to a better performance from the model which is also closer to reality.

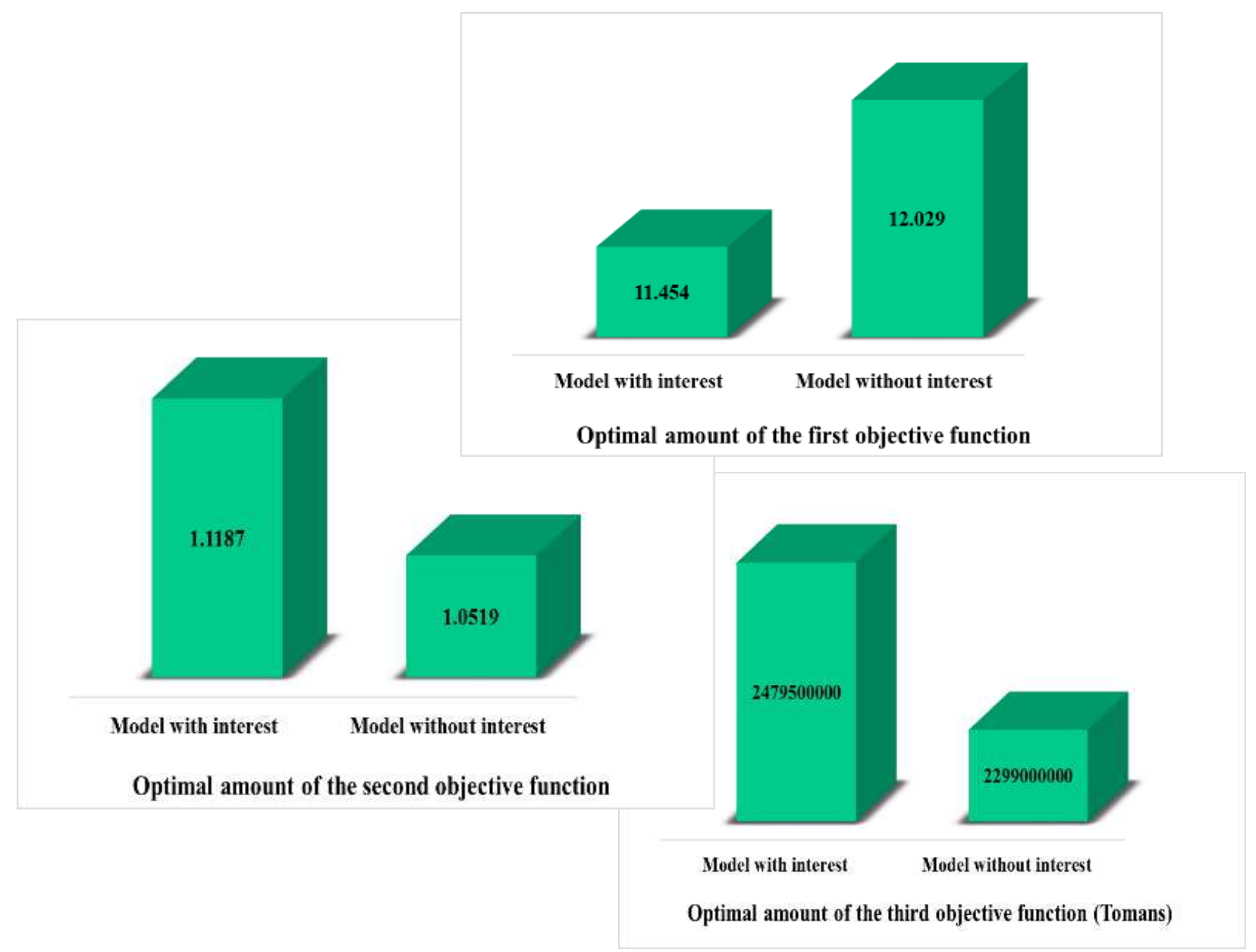

Fig. 12. The results obtained from evaluating the effects of bank interest rate on the proposed model. 
2.3. Evaluating the performance of the multi-period optimization approach: HolguínVeras et al. (2013) asserted that the inter-temporal effects of humanitarian relief logistics activities cannot be considered in single-period optimization. Therefore, in this paper, it is claimed that integrating the main decisions such as location, procurement, and distribution in a multi-period horizon may improve coordination in the humanitarian relief supply chain. In order to substantiate this claim, the model was first formulated as a single-period model; then, for each period, this single-period model was solved separately; the obtained outcomes are shown in Table 13. Regarding the last column in Table 13, it is observed that the multi-period optimization model (proposed model) outperforms the single-period optimization model in responding to the affected people and has a more affordable total cost. Therefore, the multi-period optimization model outperforms the single-period optimization model.

2.4. Investigating the effects of gradual budget injection into the system: To examine the performance of the proposed approach to injecting budgets into the system (the gradual budget injection approach), it is first assumed that the total budget planned for the establishment of warehouses and the total budget planned for the procurement of commodities at the beginning of the planning time horizon are fully available (the instantaneous budget injection approach). Subsequently, the amounts of budgets for each period are considered as follows:

$$
B_{1}=\mathrm{B} ; B_{2}=0 ; B_{3}=0 ; A_{1}=\mathrm{A} ; A_{2}=0 ; A_{3}=0
$$

Next, the model was solved according to these budget values, the results of which are presented in Table 14.

It is not possible to implement the decisions presented in Table 14 in due time since at the beginning of the planning time horizon, establishment and procurement budgets are not fully available; rather, at the beginning of each period, a part of the budgets are injected into the project. Therefore, to schedule for establishing the selected warehouses, procure the determined amounts of relief packages, and set the optimal strategy for distributing relief packages among the demand points, the model is solved according to the solutions presented in Table 14 and the actual amount of budgets at each period. Given the increasing establishment and procurement costs over time, it is not possible to establish all of the selected warehouses and procure all of the determined relief packages; as a result, the model is not solved. If one of the warehouses is not built, then the model can be solved. Therefore, not establishing warehouse 8 compared to not establishing other selected

Table 13. Results obtained from the single-period optimization model.

\begin{tabular}{lcccc}
\hline \multicolumn{1}{c}{ period } & $\mathbf{1}$ & $\mathbf{2}$ & $\mathbf{3}$ & Sum \\
\hline Optimal amount of the second objective function & 0.23682 & 0.36952 & 0.46955 & 1.07589 \\
\hline Optimal amount of the third objective function (Tomans) & $5.8293 \mathrm{E}+8$ & $8.4314 \mathrm{E}+8$ & $1.1312 \mathrm{E}+9$ & $2.55727 \mathrm{E}+9$ \\
\hline
\end{tabular}

Table 14. Selected warehouses along with their specifications and the amount of storage of relief items within them using the model with instantaneous budget injection.

\begin{tabular}{ccccccc}
\hline Warehouse & $\begin{array}{c}\text { Establishment } \\
\text { time }\end{array}$ & $\begin{array}{c}\text { Storage } \\
\text { level }\end{array}$ & $\begin{array}{c}\text { Retrofitting } \\
\text { level }\end{array}$ & \multicolumn{3}{c}{ Stocked packages } \\
\cline { 5 - 7 } & 1 & Large & High & 72000 & period 2 & period 3 \\
\hline 2 & 1 & Large & Medium & 72000 & 0 & 0 \\
\hline 5 & 1 & Large & Medium & 72000 & 0 & 0 \\
\hline 6 & 1 & Small & Medium & 30000 & 0 & 0 \\
\hline 7 & 1 & Medium & Medium & 48000 & 0 & 0 \\
\hline 8 & 1 & Medium & High & 36718 & 0 & 0 \\
\hline 10 & & & & & & \\
\hline
\end{tabular}


warehouses leads to better results for the first and second objective functions, which also have a higher priority for the relief organization. In Fig. 13, the approach presented in this evaluation for injecting budgets into the system (instantaneous budget injection approach) is compared with the proposed gradual budget injection approach. According to Fig. 13, the gradual budget injection approach led to a more efficient response to affected people, more cost-effective total cost, and more desirable warehouses based on the input and output criteria.

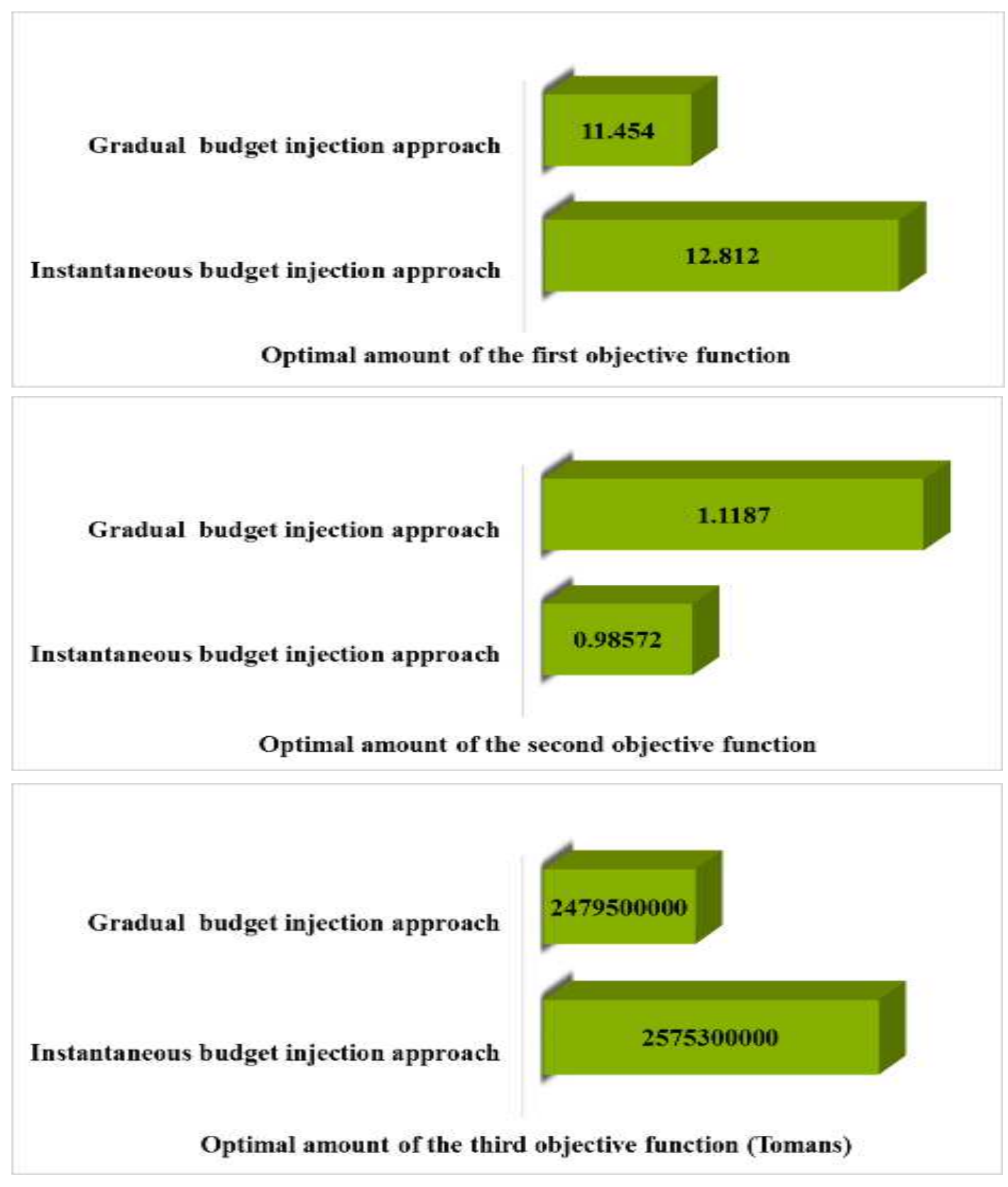

Fig. 13. Comparison of the instantaneous budget injection approach with the gradual budget injection approach. 
In order to establish warehouse 8 and store the determined amount of relief packages in it, the establishment and procurement budgets for each period are increased. Therefore, the objective functions reach the values of 11.954, 1.0926, and 3006800000, respectively. Consequently, according to the values of the objective functions, it is observed that the full implementation of location and procurement decisions presented in Table 14 does not still lead to better performance of the relief network.

According to the results presented above, it can be concluded that the gradual budget injection approach involves a better performance.

\section{2. Robustness analysis}

Expected value of perfect information (EVPI) is a performance measure that represents the value of access to accurate information about the future and somehow expresses the acceptable cost of access to more accurate information. EVPI is calculated as follows:

$\mathrm{WSS}=\sum_{S} p_{S} z_{S}$

$\mathrm{EVPI}=\mathrm{RP}-\mathrm{WSS}$ (for minimization problems) or WSS-RP (for maximization problems)

Where $z_{S}$ and RP represent the optimal value of the objective function of the deterministic (single-scenario) problem associated with scenario $s$ and the optimal value of the objective function of the original problem, respectively. Higher EVPI usually represents the considerable impact of randomness on a given problem (Birge and Louveaux, 1997). The results obtained from calculating EVPI for the proposed model are presented in Table 15.

Regarding the outcomes presented in Table 15, it is observed that the planning of the proposed problem is very close to that of the deterministic problem associated with each scenario. Therefore, very small values of EVPI indicate the importance of using scenario-based stochastic programming in location, procurement, and distribution decisions and the important role of randomness in the proposed model.

Table 15. Results obtained from robustness analysis

\begin{tabular}{ccccc}
\hline & $\boldsymbol{Z}_{\mathbf{1}}^{*}$ & $\boldsymbol{Z}_{\mathbf{2}}^{*}$ & $\boldsymbol{Z}_{\mathbf{3}}^{*}$ & $\boldsymbol{\lambda}$ \\
\hline Scenario 1 & 11.441 & 1.212 & 2499000000 & 1.6666 \\
\hline Scenario 2 & 11.447 & 1.1593 & 2516600000 & 1.6656 \\
\hline Scenario 3 & 11.451 & 0.9409 & 2456700000 & 1.6651 \\
\hline Scenario 4 & 11.443 & 1.0248 & 2516400000 & 1.6663 \\
\hline WSS & 11.4441 & 1.1418 & 2592660000 & 1.6661 \\
\hline RP & 11.454 & 1.1187 & 2479500000 & 1.6647 \\
\hline EVPI & 0.0099 & 0.0231 & -23160000 & 0.0014 \\
\hline
\end{tabular}

\section{Conclusion}

Natural and manmade disasters kill thousands of people and displace millions every year. Therefore, to decrease the damages caused by disasters, proper planning is essential in dealing with these events before their occurrence. Consequently, this study aimed at presenting a logistical model for simultaneous strategic decision-making with respect to humanitarian relief logistics planning (warehouse location for storage of relief items along with determining their capacity/size, retrofitting level, and the amount of storage of relief items in each warehouse pre-disaster) and 
certain operational decisions (how to give out relief items among the disaster victims postdisaster). In this regard, a stochastic multi-objective mixed-integer non-linear programming model was presented to minimize the total social cost and maximize fulfilled demands and warehouse utility. The maximization of warehouse utility was formulated in the proposed mathematical programming model according to a proposed DEA model based on the DEA model introduced by Sun et al. (2013). Moreover, the maximization of fulfilled demands and the minimization of deprivation costs considering equitable response (distribution) were simultaneously calculated using a new objective function based upon the fraction of satisfied demand, the reverse of the deprivation cost function, and the priority of demand point. In this study, several pre-disaster periods, a limited budget for establishing warehouses and procuring relief items with gradual budget injection into the system, the time value of money, various criteria for evaluating warehouses, and the risk of disruption in warehouses, transportation networks, and heterogeneous warehouses $\mathrm{h}$ were taken into account. It is worth noting that, location, procurement, and distribution problems under discussion were modeled dynamically (time-dependent) due to the assumed financial constraints. Finally, the evaluation and validation of the proposed programming model were performed using a numerical example close to real conditions; subsequently, the following results were obtained:

- The proposed DEA model resulted in a more efficient relief network compared to certain other DEA models as well as its combination with the mathematical programming model compared to its non-integration state.

- Minimizing the weighted sum of the sum of utility scores of the established warehouses and the maximum of these scores yielded more desirable results compared to the sole minimization of the sum of utility scores of the established warehouses,.

- The considered deprivation cost function outperforms certain other functions presented in the literature on humanitarian relief logistics.

- Consideration of the time value of money in the form of bank interest allocated to the remaining establishment and procurement budgets at the end of a period, the proposed gradual budget injection approach, and the multi-period optimization approach increased the efficiency of the relief network performance.

- The proposed model involves sufficient robustness.

No research can be fully comprehensive and complete and not all dimensions can be examined in a single study. Besides, researchers gain valuable information during the research process that can be useful for other scholars. Accordingly, the development of the problem in question can be suggested to other researchers for future inquiries. To this end, the following points can be taken into account:

- The random and unpredictable nature of the crisis necessitates crisis management within an uncertain environment. As a result, many of the examined papers used a scenario-based stochastic optimization approach while other uncertain optimization approaches were overlooked. Besides, given the researchers' identification of disadvantages in the scenariobased stochastic programming approach presenting an uncertain optimization approach such as robust optimization, fuzzy optimization, probabilistic optimization, etc. for the problem under examination along with an efficient solution algorithm can be useful.

- To provide excellent levels of service, processes in a humanitarian relief supply chain should be analyzed in general, rather than in detail. As a result, decentralized and hierarchical decisions can better achieve the goals of a humanitarian relief supply chain. For this purpose, the use of multi-level programming can be beneficial. Nevertheless, very few studies have focused on 
multi-level optimization problems to date. Therefore, modeling the problem under study in the form of a multi-level optimization model can provide a more realistic relief network; it also allows for the observation of how decisions made in each part of the network can influence or be influenced by decisions made in other areas.

- Certain vital relief items such as food, water, medicine, etc. are perishable; as a result, lack of attention to their corruption can lead to considerable financial and human losses. Subsequently, it is necessary to consider corrupted relief items in the proposed problem and provide an efficient inventory management strategy.

- To decrease the expenses of procuring relief goods and the risk of supplying relief goods after a disaster, it is necessary to select appropriate suppliers and provide solutions to deal with the risk of supply disruption caused by disruptive suppliers. Moreover, the procurement of relief items using a strategy based on establishing contracts with supply sources can decrease the expenses of procurement and warehousing due to fewer prepositioned items in inventory and lower supply risk. Hence, the responsiveness and cost efficiency of the proposed humanitarian relief chain can be enhanced by integrating the problem under discussion with a supplier selection problem, in which a new and realistic strategy is used based upon establishing contracts with suppliers; solutions would also be offered to deal with the risk of supplier disruption.

- To solve the proposed model in a large-scale and implement it in real cases, presenting an exact solution method or a heuristic/meta-heuristic algorithm and surveying real cases are also recommended.

\section{Compliance with ethical standards}

Conflict of interest: The authors declare that they have no conflict of interest.

Research involving human participants and/or animal: This article does not contain any studies with human participants or animals performed by any of the authors.

Informed consent: Informed consent was obtained from all individual participants included in the study.

\section{Reference}

Afsharian, M., 2019. A frontier-based facility location problem with a centralised view of measuring the performance of the network. Journal of the Operational Research Society, DOI: 10.1080/01605682.2019.1639476, 1-16. (In press)

Aghajani, M., Torabi, S. A., Heydari, J., 2020. A novel option contract integrated with supplier selection and inventory prepositioning for humanitarian relief supply chains. Socio - Economic Planning Sciences, DOI: 10.1016/j.seps.2019.100780. (In press)

Altay, N., Green Iii, W. G., 2006. OR / MS research in disaster operations management. European Journal of Operational Research, 175 (1), 475-493.

Balcik, B., Ak, D., 2014. Supplier selection for framework agreements in humanitarian relief. Production and Operations Management, 23 (6), 1028-1041. 
Balcik, B., Beamon, B. M., 2008. Facility location in humanitarian relief. International Journal of Logistics, 11 (2), 101-121.

Beamon, B. M., Balcik, B., 2008. Performance measurement in humanitarian relief chains. International Journal of Public Sector Management, 21, 4-25.

Bell, M. G. H., Fonzone, A., Polyzoni, C., 2014. Depot location in degradable transport networks. Transportation Research Part B, 66, 148-161.

Birge, J. R., Louveaux, F., 1997. Introduction to stochastic programming. Springer, NewYork.

Bozorgi-Amiri, A., Khorsi, M., 2016. A dynamic multi-objective location-routing model for relief logistic planning under uncertainty on demand, travel time, and cost parameters. Int J Adv Manuf Technol, 85, 1633-1648.

Braveman, P., Gruskin, S., 2003. Defining equity in health. J. Epidemiol Community Health, 57, 254-258.

Caunhye, M., Zhang, Y., Li, M., Nie, X., 2016. A location - routing model for prepositioning and distributing emergency supplies. Transportation Research Part E, 90, 161-176.

Chen, D., 2020. Prepositioning network design for disaster reliefs: Stochastic models and $\Psi-$ expander models comparison. Computers \& Industrial Engineering, 140, 106214 (13 pages).

Condeixa, L. D., Leiras, A., Oliveira, F., Brito, I. D., 2017. Disaster relief supply prepositioning optimization: a risk analysis via shortage mitigation. International Journal of Disaster Risk Reduction, 25, 238-247.

Cotes, N., Cantillo, V., 2019. Including deprivation costs in facility location models for humanitarian relief logistics. Socio-Economic Planning Sciences, 65, 89-100.

Daskin, M. S., Dean, L. K., 2005. Location of health care facilities. In: Operations research and health care, Springer, US, 43-76.

Elçi, Ö. Noyan, N., 2018. Chance-Constrained two-stage stochastic programming model for humanitarian relief network design. Transportation Research Part B, 108, 55-83.

Holguín-Veras, J., Amaya-Leal, J., Cantillo, V., Van Wassenhove, L. N., Aros-Vera, F., Jaller, M., 2016. Econometric estimation of deprivation cost functions: A contingent valuation experiment. Journal of Operations Management, 45, 44-56.

Holguín-Veras, J., Pérez, N., Jaller, M., Van Wassenhove, L. N., Aros-Vera, F., 2013. On the appropriate objective function for post-disaster humanitarian logistics models. Journal of Operations Management, 31 (5), 262-280.

Hu, S., Dong, Z. S., 2019. Supplier selection and pre-positioning strategy in humanitarian relief. Omega, 83 (C), 287-298.

Hwang, H. S., 2004. A stochastic set-covering location model for both ameliorating and deteriorating items. Computers \& industrial engineering, 46 (2), 313-319.

İlhan, A., 2011. The humanitarian relief chain. South East European Journal of Economics and Business, 1 (9), 15-51.

Irohara, T., Kuo, Y. H., Leung, J. M. Y., 2013. From Preparedness to Recovery: A tri-level programming model for disaster relief planning. Conference: International Conference on Computational Logistics, Copenhagen, Denmark, 213-228.

Jang, H. C., Lien, Y. N., Tsai, T. C., 2009. Rescue information system for earthquake disasters based on MANET emergency communication platform. Proceedings of the International Conference on Wireless Communications and Mobile Computing: Connecting the World Wirelessly, IWCMC 2009, Leipzig, Germany, 623-627.

Klimberg, R. K., Ratick, S. J., 2008. Modeling data envelopment analysis (DEA) efficient location/allocation decisions. Computers \& Operations Research, 35, 457-474. 
Li, X., Ramshani, M., Huang, Y., 2018. Cooperative maximal covering models for humanitarian relief chain management. Computers \& Industrial Engineering, 119, 301-308.

Liberatore, F., Ortuño, M. T., Tirado, G., Vitoriano, B., Scaparra, M. P., 2014. A hierarchical compromise model for the joint optimization of recovery operations and distribution of emergency goods in Humanitarian Logistics. Computers \& Operations Research, 42, 3-13.

Lin, C. C., 2004. A weighted max-min model for fuzzy goal programming. Fuzzy Sets and Systems, 142 (3), 407-420.

Lu, C., 2013. Robust weighted vertex p - center model considering uncertain data: An application to emergency management. European Journal of Operational Research, 230, 113-121.

Lu, C., Sheu, J. B., 2013. Robust vertex p - center model for locating urgent relief distribution centers. Computers \& Operations Research, 40, 2128-2137.

Mete, O., Zabinsky, Z. B., 2010Stochastic optimization of medical supply location and distribution in disaster management. Int. J. Production Economics, 126, 76-84.

Mohamadi, A., Yaghoubi, S., 2017. A bi-objective stochastic model for emergency medical services network design with backup services for disasters under disruptions: An earthquake case study. International Journal of Disaster Risk Reduction, 23, 204-217.

Mohamadi, A., Yaghoubi, S., Pishvaee, M. S., 2019. Fuzzy multi-objective stochastic programming model for disaster relief logistics considering telecommunication infrastructures: a case study. Operational Research, 19 (1), 59-99.

Mulvey, J. M., Vanderbei, R. J., Zenios, S. A., 1995. Robust optimization of large-scale systems. Oper. Res., 43 (2), 264-281.

Noham, R., Tzur, M., 2018. Designing humanitarian supply chains by incorporating actual postdisaster decisions. European Journal of Operational Research, 265 (3), 1064-1077,

Noyan, N., Merakl1, M., Küçükyavuz, S., 2019. Two-stage stochastic programming under multivariate risk constraints with an application to humanitarian relief network design. Mathematical Programming, https://doi.org/10.1007/s10107-019-01373-4, 1-39.

Rahmani, D., Zandi, A., Peyghaleh, E., Siamakmanesh, N., 2018. A robust model for a humanitarian relief network with backup covering under disruptions: A real world application. International Journal of Disaster Risk Reduction, 28, 56-68.

Ransikarbum, K., Mason, S., 2016. Goal programming-based post-disaster decision making for integrated relief distribution and early-stage network restoration. Int. J. Production Economics, $182,324-341$.

Rath, S., Gutjahr, W. J., 2014. A math-heuristic for the warehouse location-routing problem in disaster relief. Computers \& Operations Research, 42, 25-39.

Renklia, C., Duran, S., 2015. Pre - positioning disaster response facilities and relief items. Human and Ecological Risk Assessment: An International Journal, 21 (5), 1169-185.

Rezaei-Malek, M., Tavakkoli-Moghaddam, R., Cheikhrouhou, N., Taheri-Moghaddam, A. R., 2016 (a). An approximation approach to a trade - off among efficiency, efficacy, and balance for relief pre - positioning in disaster management. Transportation Research Part E, 93, 485509.

Rezaei-Malek, M., Tavakkoli-Moghaddam, R., Zahiri, B., Bozorgi-Amiri, A., 2016 (b). An interactive approach for designing a robust disaster relief logistics network with perishable commodities. Computers \& Industrial Engineering, 94, 201-215.

Salman, S. Yücel, E., 2015. Emergency facility location under random network damage: Insights from the Istanbul case. Computers \& Operations Research, 62, 266 - 281. 
Sun, J., Wu, J., Guo, D., 2013. Performance ranking of units considering ideal and anti-ideal DMU with common weights. Applied Mathematical Modelling, 37, 6301-6310.

Tavana, M., Abtahi, A. R., Di Caprio, D., Hashemi, R., Yousefi-Zenouz, R., 2018. An integrated location-inventory-routing humanitarian supply chain network with pre - and post - disaster management considerations. Socio-Economic Planning Sciences, 64, 21-37.

Thomas, A. S., Kopczak, L. R., 2005. From logistics to supply chain management: the path forward in the humanitarian sector. Fritz Institute, 65, 6-65.

Tomasini, R., Van Wassenhove, L. N., 2009. Humanitarian logistics. Houndmills, Basingstoke; New York, NY: Palgrave Macmillan.

Torabi, S. A., Shokr, I., Tofighi, S., Heydari, J., 2018. Integrated relief pre-positioning and procurement planning in humanitarian supply chains. Transportation Research Part E, 113, $123-146$.

Van Hentenryck, P., Bent, R., Coffrin, C., 2010. Strategic Planning for Disaster Recovery with Stochastic Last Mile Distribution. CPAIOR: International Conference on Integration of Artificial Intelligence (AI) and Operations Research (OR) Techniques in Constraint Programming, Bologna, Italy, 318-333.

Wang, B. C., Li, M., Hu, Y., Huang, L., Lin, S. M., 2020. Optimizing locations and scales of emergency warehouses based on damage scenarios. Journal of the Operations Research Society of China, 8, 437-456.

Wang, Q., Nie, X., 2019. A stochastic programming model for emergency supply planning considering traffic congestion. IISE Transactions, 51 (8), 910 - 920.

Wojcik, V., Dyckhoff, H., Clermont, M., 2019. Is data envelopment analysis a suitable tool for performance measurement and benchmarking in non-production contexts?. Business Research, $12(2), 559-595$.

Yu, C. S., Li, H. L., 2000. A robust optimization model for stochastic logistic problems. International Journal of Production Economics, 64 (1 - 3), 385-397.

Zhang, J., Liu, H., Yu, G., Ruan, J., Chan, F. T. S., 2019. A three-stage and multi-objective stochastic programming model to improve the sustainable rescue ability by considering secondary disasters in emergency logistics. Computers \& Industrial Engineering, 135, 11451154.

\section{Appendix}

The proposed programming model based on the DEA model employed in the research conducted by Klimberg and Ratick (2008) is as follows.

$\operatorname{Max} Z_{1}=\sum_{i \in I}\left(1-u_{i}\right)$

$\operatorname{Max} Z_{2}, \operatorname{Min} Z_{3}$

S. T. :

Eqs. (5) - (7) , (11) - (15) and (17) - (19)

$\sum_{n \in N} \vartheta_{n i} E_{n i}=\sum_{l \in L} \sum_{r \in R} \sum_{t \in T} x_{i l r t} \quad \forall i \in I$

$\sum_{m \in M} \tau_{m i} O_{m i}+u_{i}=\sum_{l \in L} \sum_{r \in R} \sum_{t \in T} x_{i l r t} \quad \forall i \in I$

$\sum_{m \in M} \tau_{m i} O_{m i}-\sum_{n \in N} \vartheta_{n i} E_{n i} \leq 0$

$\forall i, i \in I ; i \neq i$ 
Where variables $u_{i}, \tau_{m i}, \vartheta_{n i}$ indicate the level of inefficiency of warehouse $i$, the weight allocated to the $m^{\text {th }}$ output criterion for warehouse $\mathrm{i}$, and the weight allocated to the $n^{\text {th }}$ input criterion for warehouse $i$, respectively. Eq. (51) maximizes the summation of the efficiencies of warehouses. Eq. (52) considers the weighted sum of the input criteria of the established and unestablished warehouse to be equal to one and zero, respectively. Eq. (53) indicates the level of inefficiency of the warehouse. Eq. (54) determines that the weighted sum of output criteria is less than or equal to the weighted sum of input criteria. Eq. (55) requires the weighted output criteria to be less than or equal to 1. Eqs. (56) and (57) express the range of the variables.

* The proposed programming model based on the DEA model used in the research conducted by Afsharian (2019) is as follows.

$\operatorname{Max} Z_{l}=\sum_{m \in M} \sum_{i \in I} \sum_{l \in L} \sum_{r \in R} \sum_{t \in T} x_{i l r t} \tau_{m} O_{m i}$

$\operatorname{Max} Z_{2}, \operatorname{Min} Z_{3}$

S. T. :

Eqs. (5) - (8) and (11) - (19)

$\sum_{n \in N} \sum_{i \in I} \sum_{l \in L} \sum_{r \in R} \sum_{t \in T} x_{i l r t} \vartheta_{n} E_{n i}=1 \quad \forall i \in I$

Eq. (58) maximizes the weighted sum of the output criteria of warehouses. Eq. (59) considers the weighted sum of the input criteria of an established warehouse to be equal to one.

The proposed DEA model according to the DEA model introduced by Sun et al. (2013) is formulated as follows.

\section{Sets and indices:}

$K$ : Set of alternatives, indexed by $k$.

$N$ : Set of inputs criteria, indexed by $n$.

$M$ : Set of outputs criteria, indexed by $m$.

\section{Parameters:}

$\omega_{n k}$ : Amount of input criterion $n$ for alternative $k$.

$\varphi_{m k}$ : Amount of output criterion $m$ for alternative $k$.

$\omega_{\text {nIDEAL }}$ : Amount of input criterion $n$ for virtual ideal alternative; $\omega_{n I D E A L}=\min _{k}\left\{\omega_{n k}\right\}$

$\varphi_{\text {mIDEAL }}$ : Amount of output criterion $m$ for virtual ideal alternative; $\varphi_{m I D E A L}=\max _{k}\left\{\varphi_{m k}\right\}$

$\alpha$ : Weight factor in the objective function.

$\operatorname{Min} Z_{l}=\alpha\left\{\sum_{k \in K}\left(\sum_{n \in N} \vartheta_{n}\left(\omega_{n k}-\omega_{n I D E A L}\right)+\sum_{m \in M} \tau_{m}\left(\varphi_{m I D E A L}-\varphi_{m k}\right)\right)\right\}+$

$(1-\alpha)\left(\max _{k \in K}\left\{\left(\sum_{n \in N} \vartheta_{n}\left(\omega_{n k}-\omega_{n I D E A L}\right)+\sum_{m \in M} \tau_{m}\left(\varphi_{m I D E A L}-\varphi_{m k}\right)\right)\right\}\right)$

$\sum_{m \in M} \tau_{m} \varphi_{m k} \leq \sum_{n \in N} \vartheta_{n} \omega_{n k} \quad \forall k \in K$ 
$\sum_{n \in N} \vartheta_{n} \omega_{n I D E A L}=1$

$\sum_{m \in M} \tau_{m} \varphi_{\text {mIDEAL }}=1$

$\vartheta_{n}, \tau_{m} \geq \varepsilon$

Eq. (60) minimizes the weighted sum of the summation of the weighted coordinate distances between the virtual ideal alternative and all alternatives and maximum weighted coordinate distances between the virtual ideal alternative and all alternatives. Eq. (61) determines that the weighted sum of output criteria is less than or equal to the weighted sum of input criteria. Eq. (62) and Eq. (63) take into account the weighted summation of the input criteria of the virtual ideal alternative and the weighted sum of the output criteria of the virtual ideal alternative equal to 1 , respectively. Finally, Eq. (64) specify the eligible domain of decision variables.

The efficiency score of the alternative $k$ is calculated as follows:

$E S_{k}=\frac{\sum_{m \in M} \tau_{m}^{*} \varphi_{m k}}{\sum_{n \in N} \vartheta_{n}^{*} \omega_{n k}}$

$\forall k \in K$

$\vartheta_{n}^{*}, \tau_{m}^{*}$ represent the optimal value of the weight assigned to the $n^{\text {th }}$ input criterion and the $m^{\text {th }}$ output criterion, respectively, which are obtained by solving the DEA model presented above. 


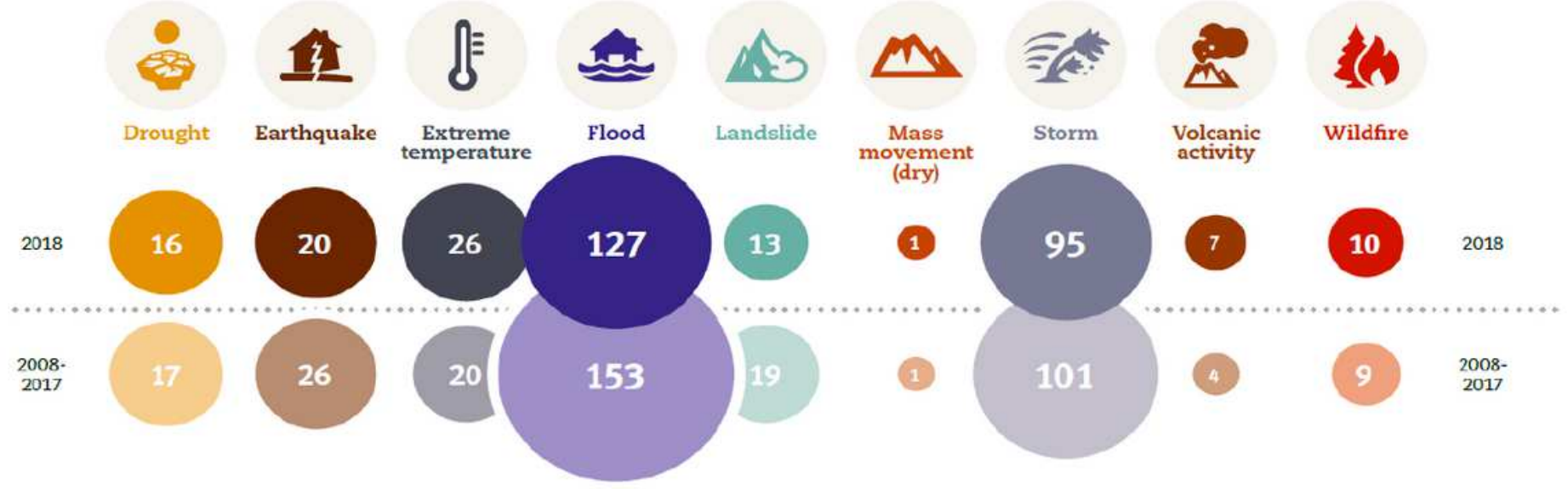

\section{Figure 1}

The number of occurrences globally by disaster type between 2008 and 2017 and in 2018 .
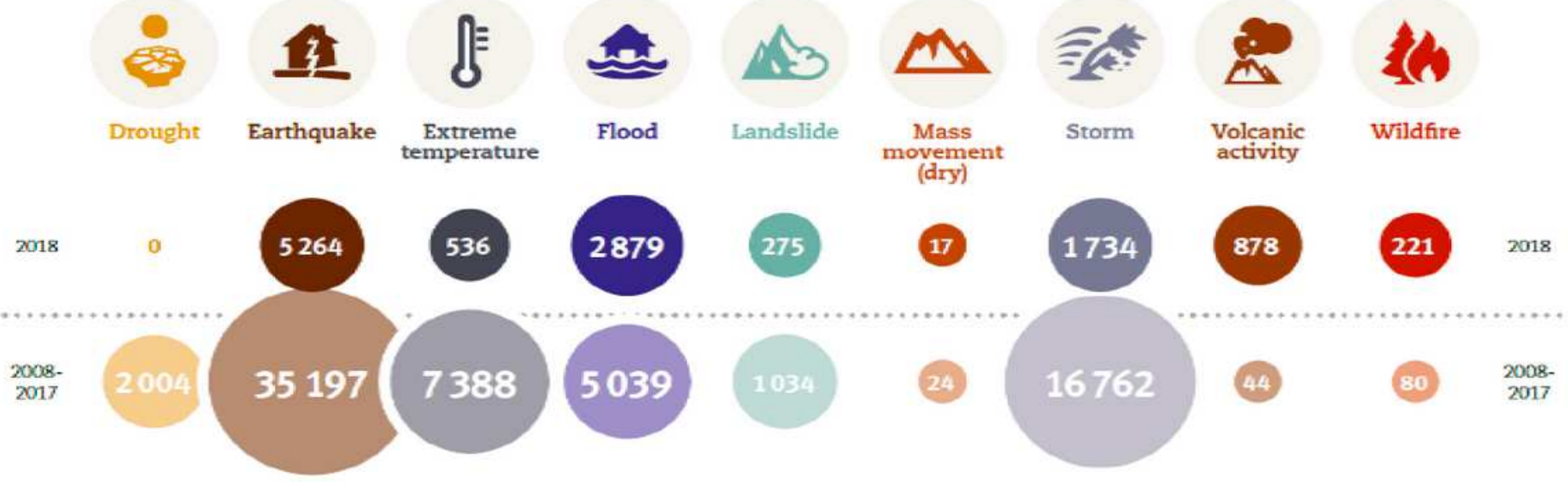

\section{Figure 2}

The number of deaths globally by disaster type between 2008 and 2017 and in 2018 .

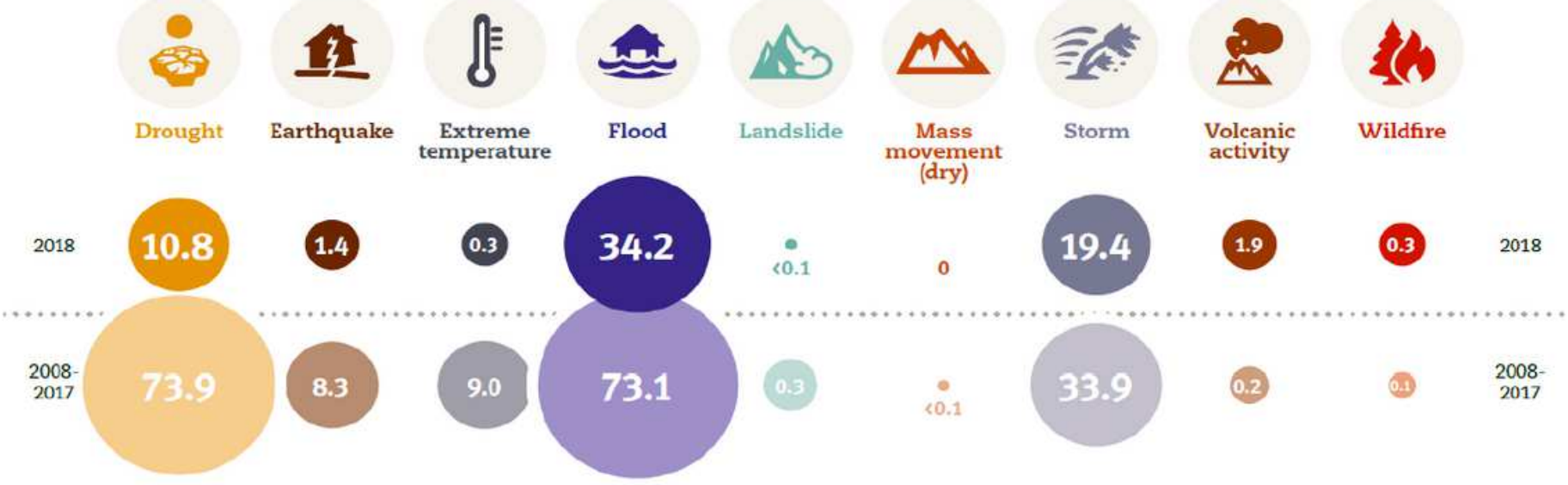


Figure 3

The number of affected (million) globally by disaster type between 2008 and 2017 and in 2018 .

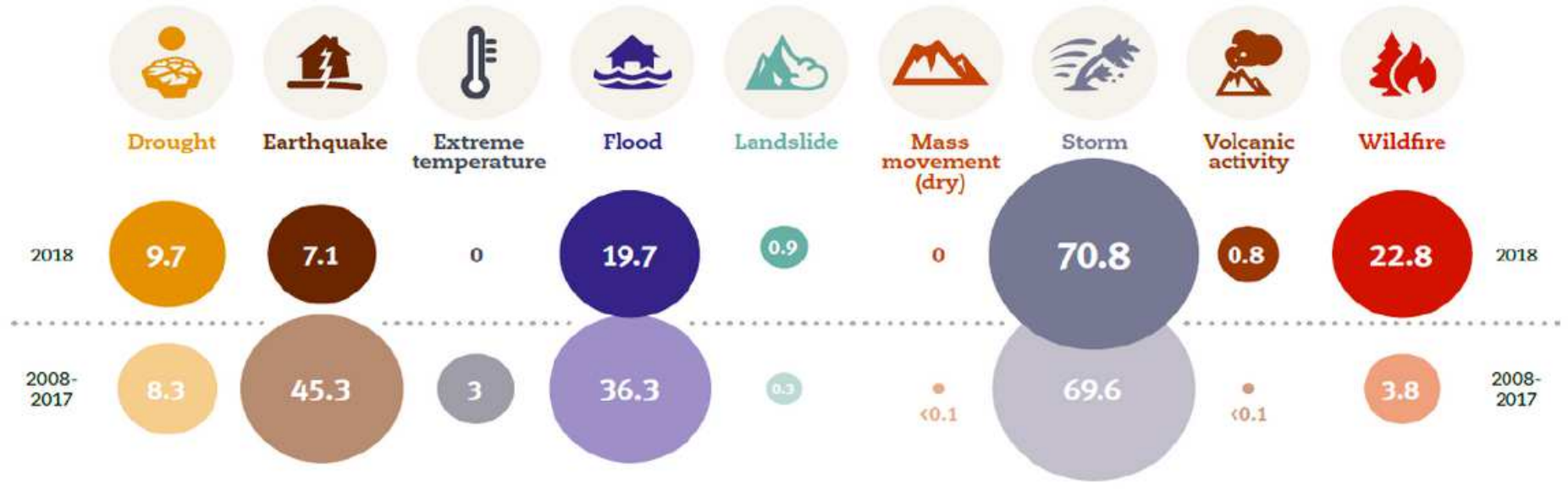

Figure 4

Economic losses (billion US\$) globally by disaster type between 2008 and 2017 and in 2018.

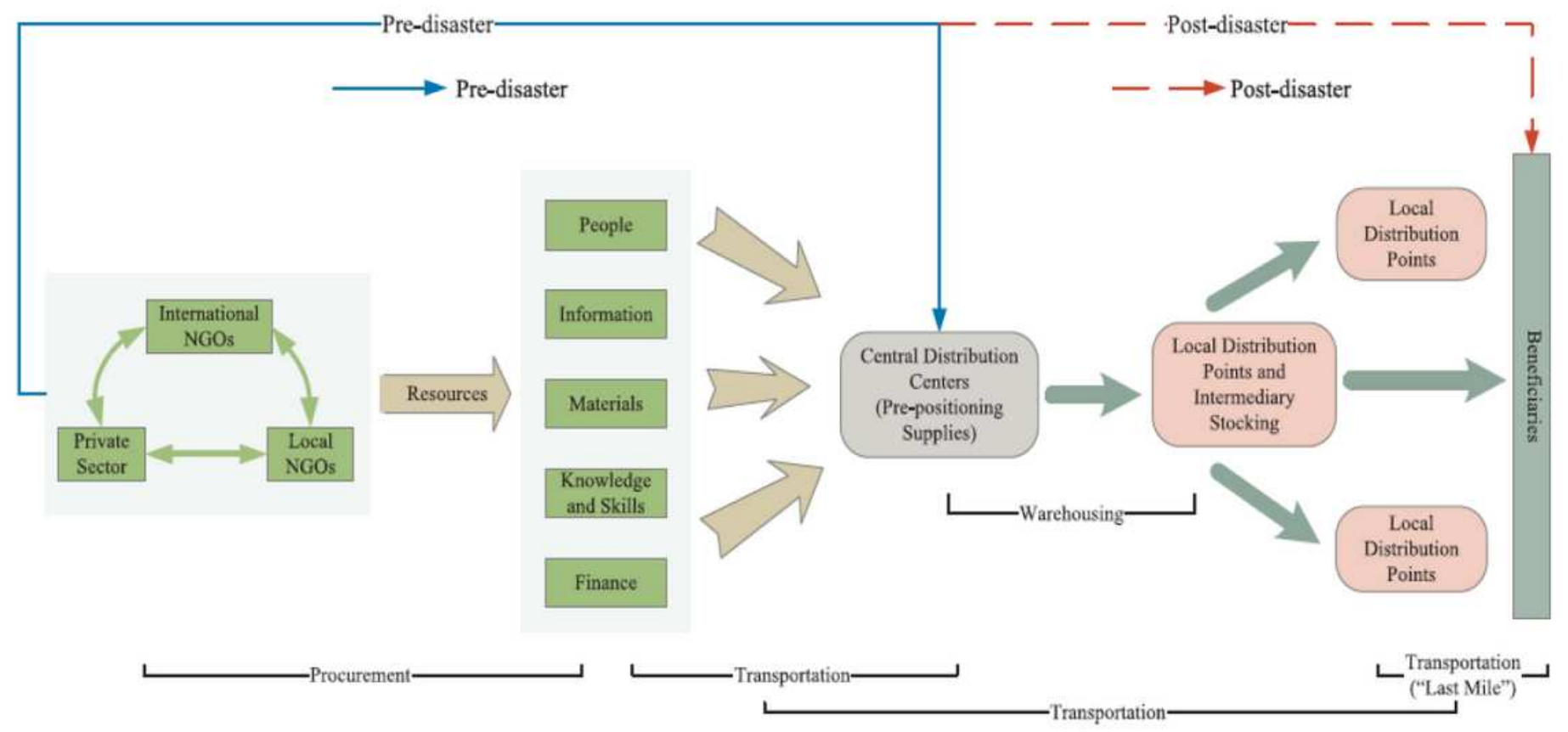

Figure 5

Humanitarian relief supply chain structure. 


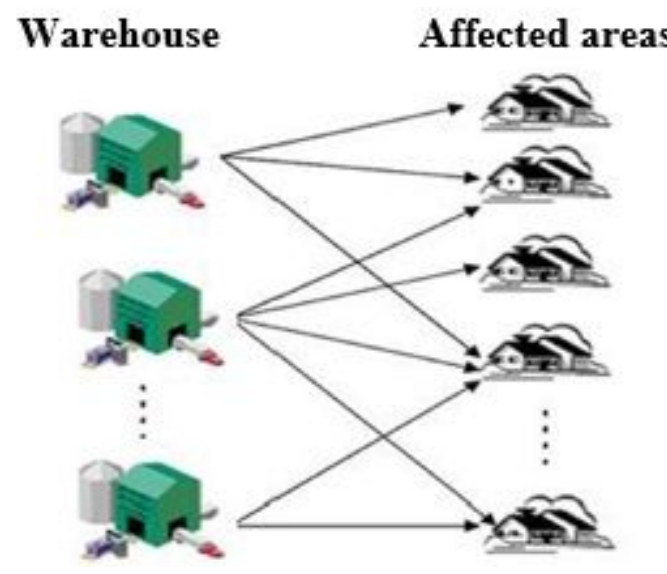

Figure 6

The general outline of the proposed humanitarian relief supply chain.
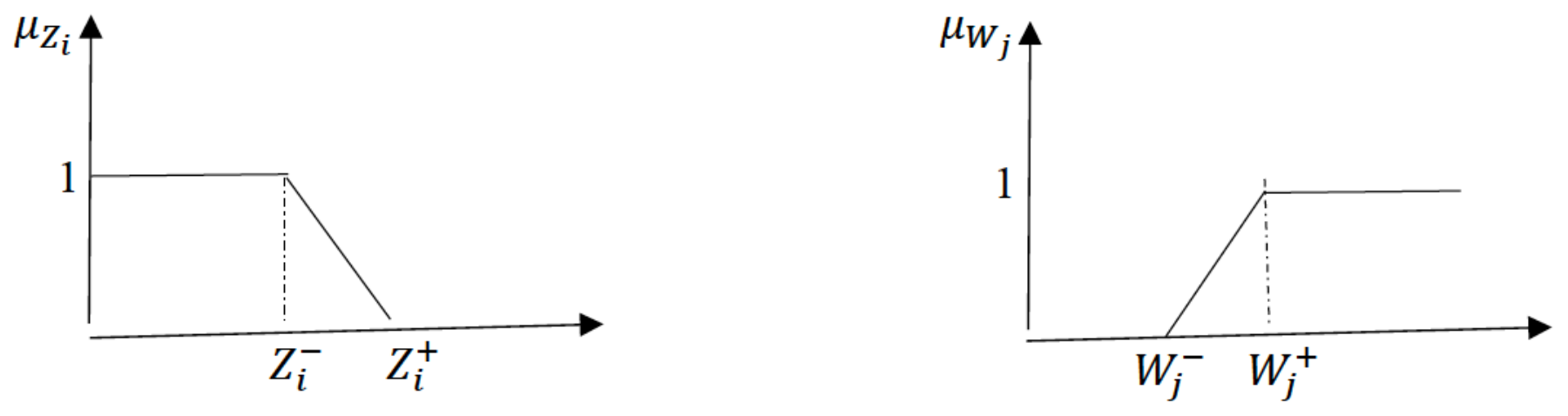

\section{Figure 7}

Membership function for objective functions.

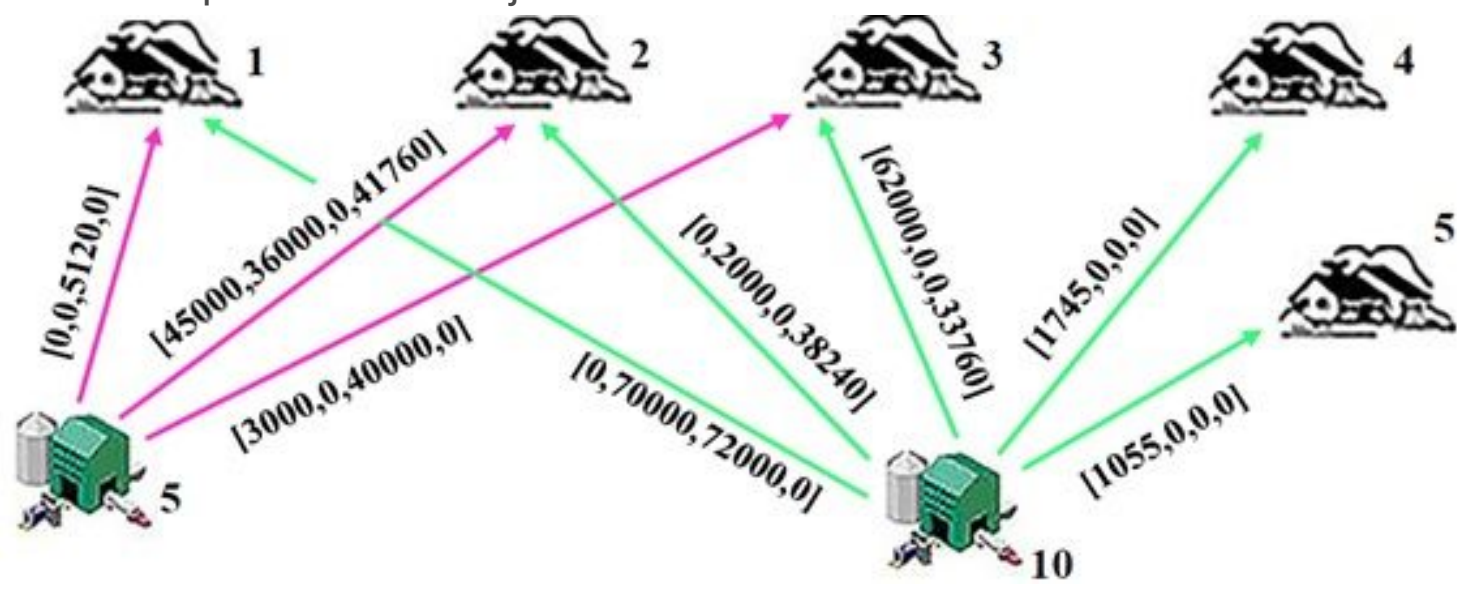

Figure 8

How relief packages are distributed among demand points at period 1 under each scenario. $\otimes$ On each arc of the network, numbers inside bracket from left to right represent the number of dispatched packages 
under the first, second, third, and fourth scenarios, respectively.

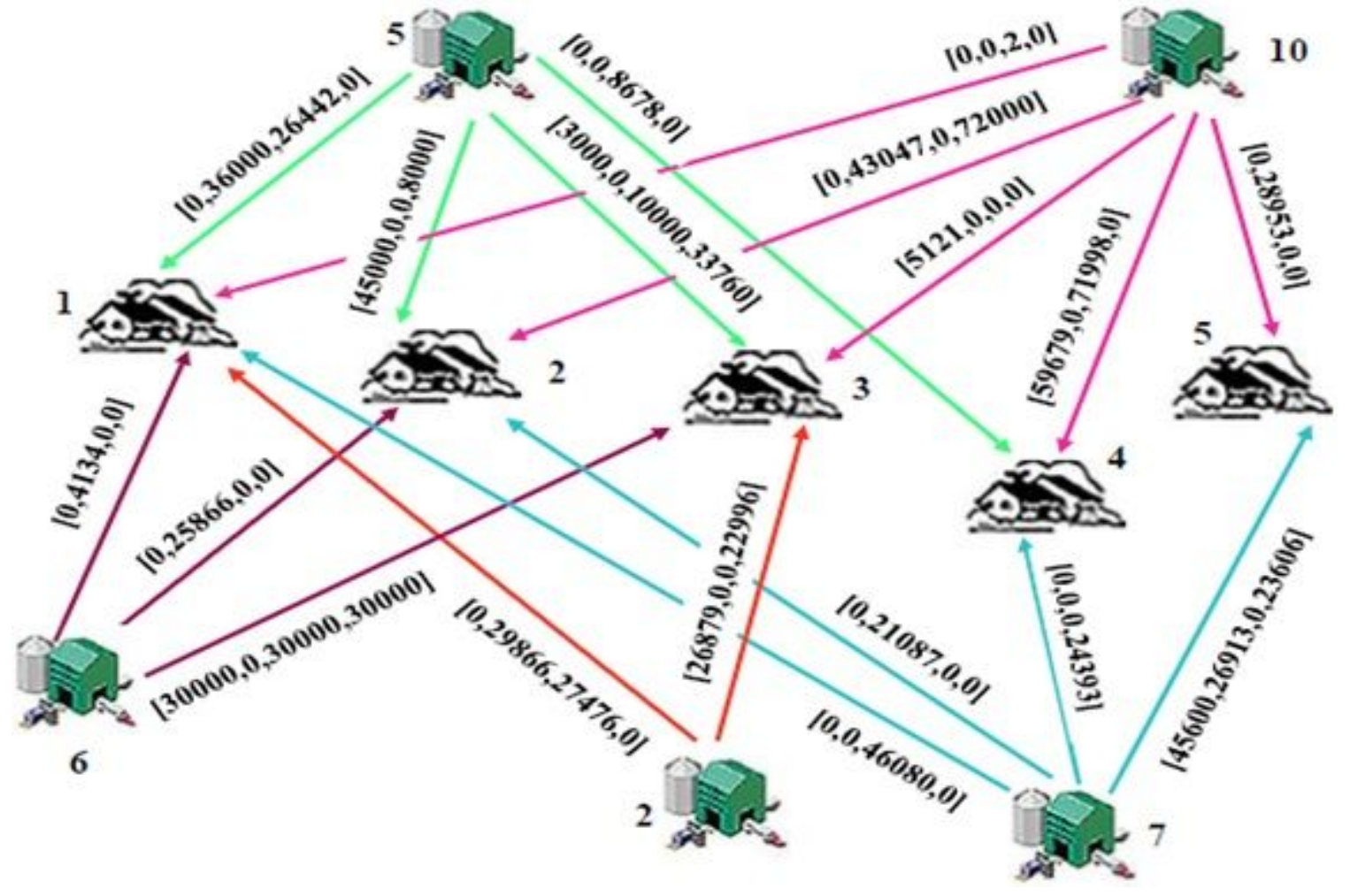

Figure 9

How relief packages are distributed among demand points at period 2 under each scenario.

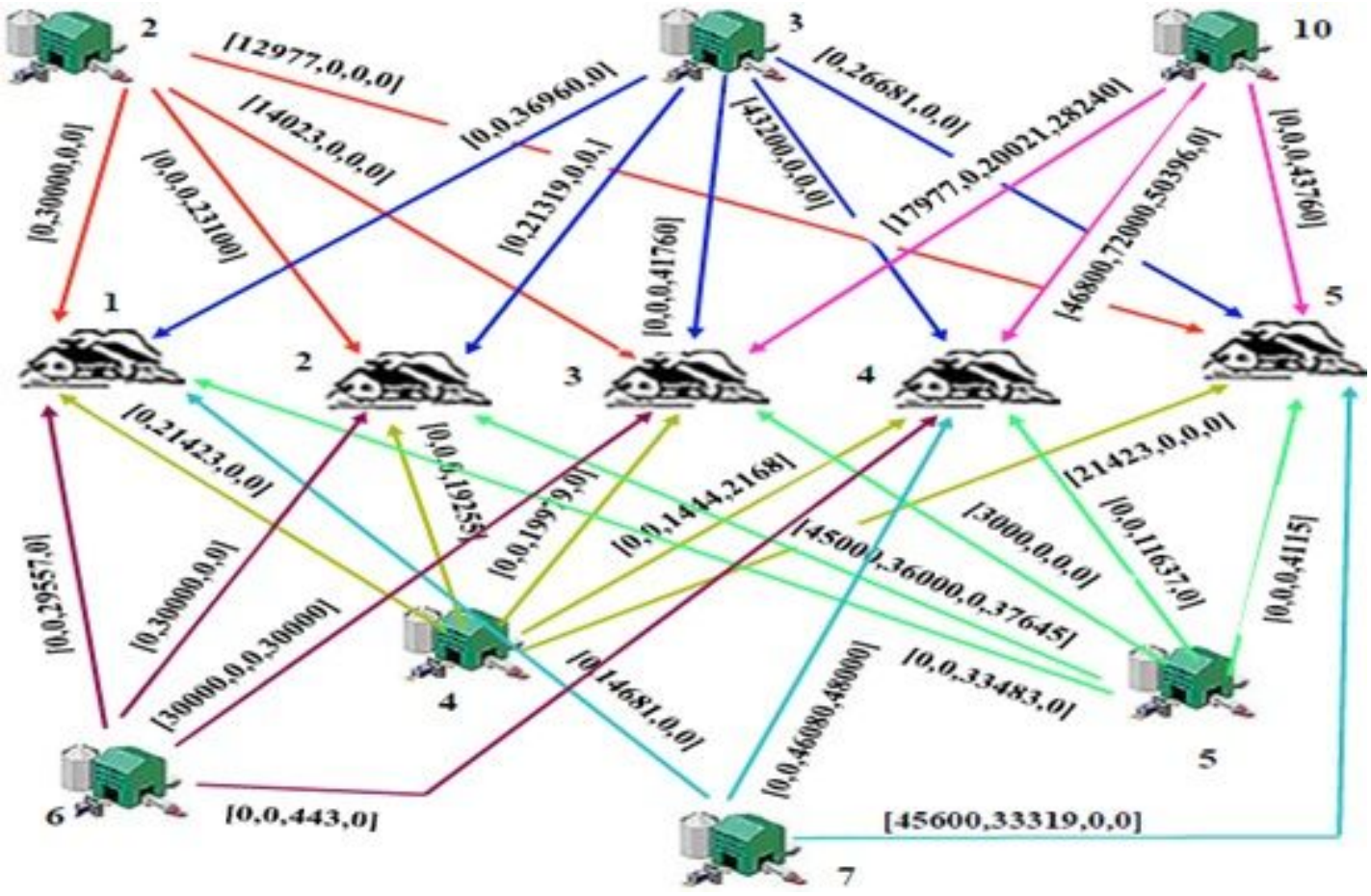

Figure 10

How relief packages are distributed among demand points at period 3 under each scenario. 


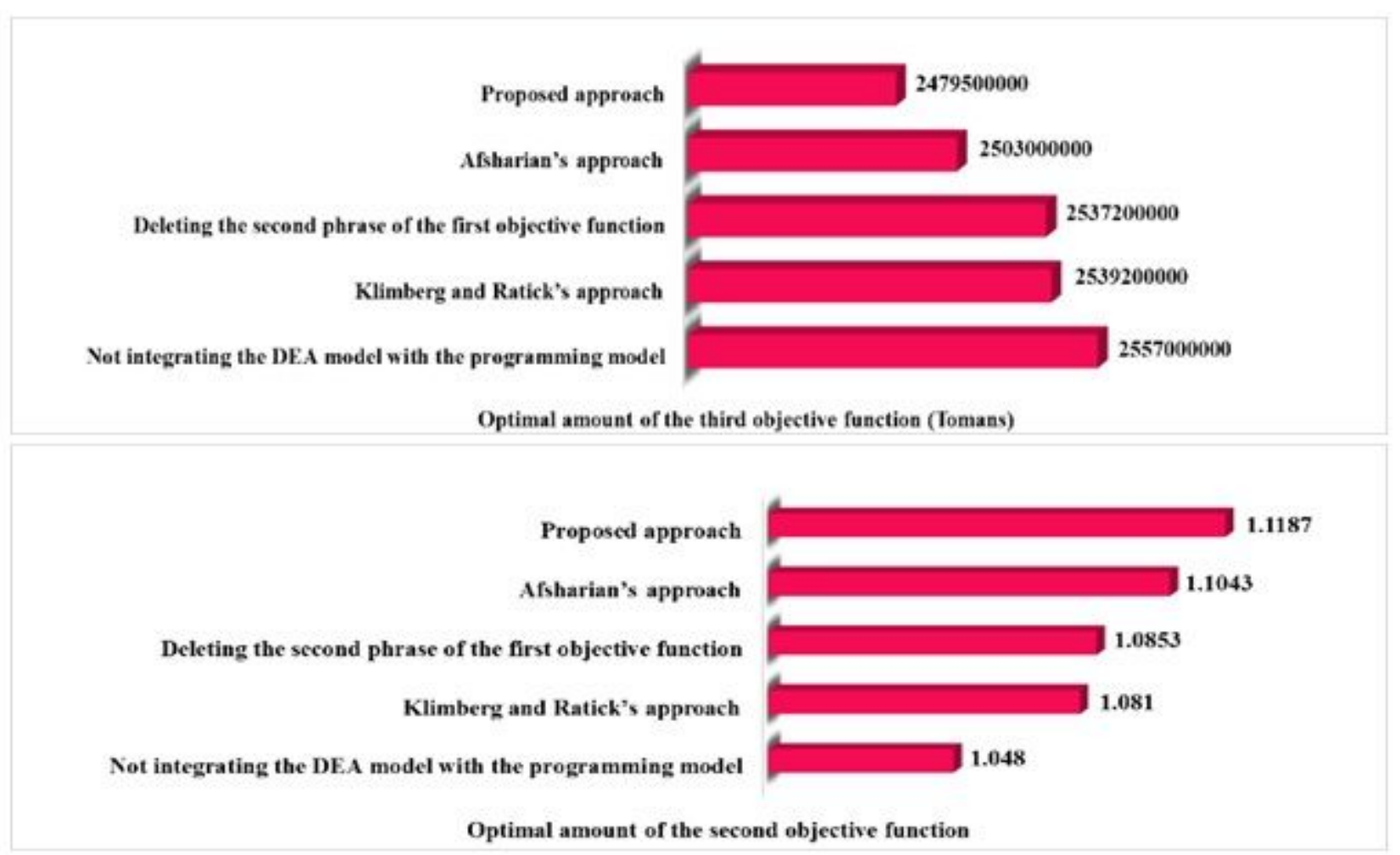

\section{Figure 11}

The results obtained from assessing the performance of the proposed procedure to establishing the most desirable warehouses according to a set of criteria. 


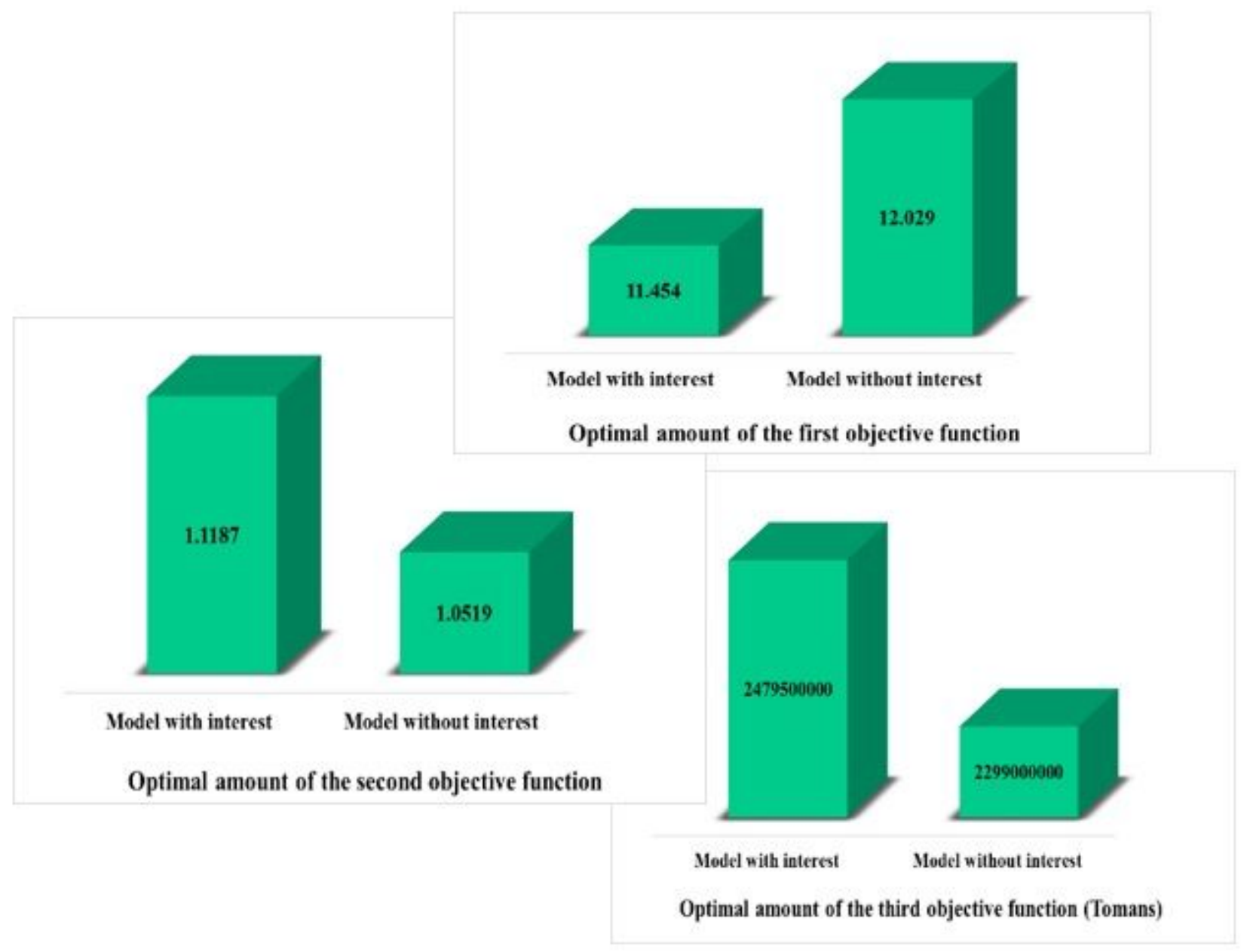

Figure 12

The results obtained from evaluating the effects of bank interest rate on the proposed model. 
Gradual budget injection approach

Instantaneous budget injection approach

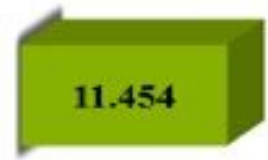

12.812

Optimal amount of the first objective function

Gradual budget injection approach

Instantaneous budget injection approach

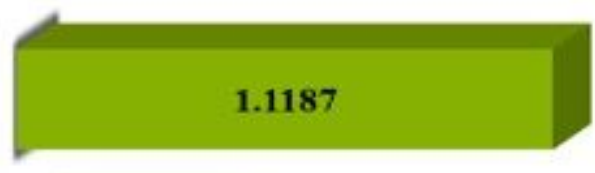

0.98572

\subsection{2 \\ (1)}

Optimal amount of the second objective function

Gradual budget injection approach

Instantaneous budget injection approach

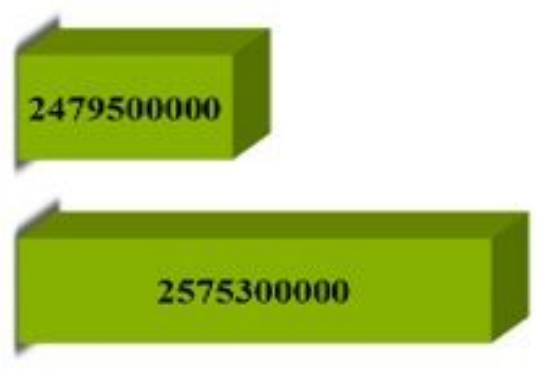

Optimal amount of the third objective function (Tomans)

\section{Figure 13}

Comparison of the instantaneous budget injection approach with the gradual budget injection approach.

\section{Supplementary Files}

This is a list of supplementary files associated with this preprint. Click to download.

- Appendix.pdf 